\title{
The Expressivity of Quantifying over Regions
}

\author{
Ernest Davis* \\ Dept. of Computer Science \\ New York University \\ davise@cs.nyu.edu
}

October 24,2011

\begin{abstract}
We categorize in recursion-theoretic terms the expressivity of a number of first-order languages that allow quantification over regions in Euclidean space. Specifically we show the following:

1. Let $\mathcal{U}$ be any class of closed regions in Euclidean space that includes all simple polygons. Let $\mathrm{C}(x, y)$ be the relation, "Region $x$ is connected to region $y$," and let Convex $(x)$ be the property, "Region $x$ is convex." Then any relation over $\mathcal{U}$ that is analytical and invariant under affine transformations is first-order definable in the structure $\langle\mathcal{U}, \mathrm{C}$, Convex $\rangle$.

2. Let $\mathcal{U}$ be as in (1), and let $\operatorname{Closer}(x, y, z)$ be the relation "Region $x$ is closer to $y$ than to $z . "$ Then any relation over $\mathcal{U}$ that is analytical and invariant under orthogonal transformations is first-order definable in the structure $\langle\mathcal{U}$, Closer $\rangle$.

3. Let $\mathcal{U}$ be the class of finite unions of intervals in the real line. Then any relation over $\mathcal{U}$ that is analytical and invariant under linear transformations is first-order definable in the structure $\langle\mathcal{U}$, Closer $\rangle$.

4. If the class of regions is restricted to be polygons with rational vertices, then results analogous to (1-3) hold, substituting "arithmetical relation" for "analytical relation".
\end{abstract}

Keywords: Expressivity, spatial representation, first-order definability, analytical relation.

\section{Introduction}

One common approach to the study of qualitative spatial representations and reasoning is to use a first-order language over a universe of spatial regions in 2- or 3-dimensional Euclidean space. For example, Gotts [9] studies the first-order language with the single predicate $\mathrm{C}(x, y)$ (Region $x$ is connected to region $y$ ); Randell, Cui, and Cohn [18] study the first-order language with the predicates $\mathrm{C}(x, y)$ and $\operatorname{Conv}(x)$ (Region $x$ is convex); and Bennett et al. [2] study the first-order language with the predicates $\mathrm{C}(x, y)$ and $\mathrm{S}(x)$ (Region $x$ is a sphere). It is often argued that by restricting the entities mentioned in the spatial language to be well-behaved, fully dimensional regions and by excluding direct references to such other geometric entities as points, lines, surfaces, angles, and so on, the resultant language will be better grounded philosophically, or more plausible cognitively, or more useful representationally.

\footnotetext{
*My thanks to Martin Davis, who pointed me toward Hinman [12] and the concept of analytical relations, and to the reviewers, who made many helpful suggestions, especially pointing out the work of Schaefer and Stefankovic [19]. This research was supported in part by NSF grants \#IIS-0097537 and \#IIS-0534809
} 
Schaefer and Stefankovic [19] have shown that the first-order language with the single predicate $\mathrm{C}(x, y)$ over the domain of closed regular regions in Euclidean space is complete for analytical relations. (Their theorem is actually stated in terms of the domain of closed curves, but it is easily shown that the two theories have equal complexity.) That is, there is an encoding that maps each integer and each set of integers into a tuple of regions in such a way that addition, multiplication, and set membership correspond to relations over regions that can be defined as firstorder formulas over the relation $\mathrm{C}(x, y)$. This result characterizes the complexity of the language but not its expressivity. For instance, it is not clear from this result whether a property like "Regions $r$ and $s$ are homeomorphic" is expressible in this language, and indeed, as far as I know, the problem is open.

Another limitation of the result is that it requires the use of regions that are "pathological" in some respects. In particular, for the proof to work, it is necessary to include pairs of regions whose boundaries intersect infinitely often (e.g. a region with the boundary $y=x \sin (1 / x)$ and a region with the boundary $y=0$.). It is not known whether the theorem holds if the universe of regions is restricted to polygons, for example, or to semi-algebraic regions. Certainly, the advocates of mereology are generally unwilling to accept such regions as legitimate; indeed the existence of such pathologies is often mentioned as a reason to reject conventional real analysis in favor of a mereological approach.

In this problem we answer the question of expressivity for a number of structures that have richer languages but allow much smaller universes of regions. (That is, as compared to the results just cited, the new results of this paper use a richer language, permit a much more restricted universe, and obtain stronger results.) Specifically, we prove the following:

1. Let $\mathcal{U}$ be any class of closed regions in Euclidean space of dimension at least 2, that includes all simple polygons. Let $\mathrm{C}(x, y)$ be the relation, "Region $x$ is connected to region $y$," and let Convex $(x)$ be the property, "Region $x$ is convex." Then any relation over $\mathcal{U}$ that is analytical and invariant under affine transformations is first-order definable in the structure $\langle\mathcal{U}, \mathrm{C}$, Convex $\rangle$. If $\mathcal{U}$ is itself analytical and invariant under affine transformations, then the converse holds: that is, a relation is first-order definable in $\langle\mathcal{U}, \mathrm{C}$, Convex $\rangle$ only if it is analytical and invariant under affine transformations.

2. Let $\mathcal{U}$ be as in (1), and let $\operatorname{Closer}(x, y, z)$ be the relation "Region $x$ is closer to $y$ than to $z$." Then any relation over $\mathcal{U}$ that is analytical and invariant under orthogonal transformations is firstorder definable in the structure $\langle\mathcal{U}$, Closer $\rangle$. If $\mathcal{U}$ is itself analytical and invariant under orthogonal transformations, then the converse holds.

3. Let $\mathcal{U}$ be the class of finite unions of intervals in the real line. Then any relation over $\mathcal{U}$ that is analytical and invariant under linear transformations is first-order definable in the structure $\langle\mathcal{U}$, Closer $\rangle$. If $\mathcal{U}$ is itself analytical and invariant under linear transformations, then the converse holds.

4. If the class of regions is restricted to be polygons with rational vertices, then results analogous to (1-3) hold, substituting "arithmetical relation" for "analytical relation".

The class of analytical relations being an extremely broad one, the above results imply quite directly that a whole host of familiar relations are definable in these languages. For example, it can easily be shown that relations such as "Regions $r$ and $s$ are homeomorphic/diffeomorphic," "Region $r$ is algebraic," "Region $r$ is a polygon with a prime number of vertices," "The area of $r$ is greater than the area of $s, "$ "The boundary of $r$ has a fractal dimension of 1.5," and so on are all analytical relations and hence are all first-order definable in structures (1) and (2) above.

The paper proceeds as follows: In section 2 , we review the definition of the class $\Delta_{\omega}^{1}$ of analytical relations, and we present a number of non-geometric structures that are complete with respect to the class. Sections 3-6 give the proofs of the above theorems. Section 7 gives some examples of 
analytical geometric relations. Section 8 reviews some related literature and section 9 suggests some related open problems.

The theorems in this paper are, as far as I know, original here, but many parts of the proof are previously known; they are reviewed here in order to make the paper somewhat self-contained. Specifically: the results of section 2 are simple consequences of recursion theory and elementary point-set topology. The results in section 3 combine results from [18], [16], and [6]. The one really new technical innovation here is the geometric definition of integers (definitions 3.29-3.34).

Notational comment: We will use lower-case symbols, such as " $x$ ", for variables, and symbols starting with a upper-case letter, such as "C" or "PointOnBoundary" for relations. We will also use standard mathematical notation for functions and relation such as $x+y$ and $x<y$. We use italicized lower case letters for variables over regions and in section 2 for variables over integers, bold-face letters such as $\mathbf{p}$ for variables over points, barred letters such as $\bar{r}$ for variables over real numbers, and hatted letters such as $\hat{s}$ for variables over infinite sequences. We follow the usual rules of precedence on Boolean operators: in decreasing order of precedence $\neg, \wedge$ and $\vee, \Rightarrow, \Leftrightarrow, \equiv$ (definitional equivalence). The scope of a quantifier is to the end of the sentence, or as indicated by brackets. Free variables are implicitly universally quantified, the scope being the entire sentence. We will sometimes use semi-colons and doubled semi-colons instead of commas as separators, e.g. "Plus $(\mathbf{a} ; \mathbf{o}, \mathbf{i} ; ; \mathbf{b} ; \mathbf{p}, \mathbf{j} ; ; \mathbf{c} ; \mathbf{r}, \mathbf{k})$ ". The purpose is just to increase legibility by grouping; logically, these semi-colons mean the same as a comma. We will use letters in calligraphic font, such as $\mathcal{L}$, or Greek letters, for meta-level symbols.

The structure with domain $\mathcal{U}$ and signature $\alpha, \beta, \gamma \ldots$ will be notated $\langle\mathcal{U}, \alpha, \beta, \gamma \ldots\rangle$.

\section{Analytical relations}

We begin by reviewing some basic definitions and results from recursion theory that we will need.

Let $\mathcal{S}_{A}$ and $\mathcal{S}_{B}$ be two structures with domains $\mathcal{U}_{A}$ and $\mathcal{U}_{B}$ respectively. An encoding of $\mathcal{S}_{B}$ in $\mathcal{S}_{A}$ is a mapping that associates each element of $\mathcal{U}_{B}$ with a $k$-tuple of elements in $\mathcal{U}_{A}$ in such a way that each of the relations in the signature of $\mathcal{S}_{B}$ corresponds to a first-order definable relation in $\mathcal{S}_{A}$. There may be $k$-tuples over $\mathcal{U}_{A}$ that do not encode any element of $\mathcal{U}_{B}$; if so, the property " $a_{1} \ldots a_{k}$ encodes an element of $\mathcal{U}_{B}$ " must be first-order definable in $\mathcal{S}_{A}$. There may be multiple $k$-tuples over $\mathcal{U}_{A}$ that encode the same element of $\mathcal{U}_{B}$; if so, the property " $a_{1} \ldots a_{k}$ encodes the same element as $a_{1}^{\prime} \ldots a_{k}^{\prime}$ " must be first-order definable in $\mathcal{S}_{A}$. We thus arrive at the following definition:

Definition 1: ${ }^{1}$ Let $\mathcal{S}_{A}$ and $\mathcal{S}_{B}$ be structures with domains $\mathcal{U}_{A}$ and $\mathcal{U}_{B}$. Let the relations $\mathcal{R}_{1} \ldots \mathcal{R}_{q}$ be the signature of $\mathcal{S}_{B}$. (A $k$-place function in the signature of $\mathcal{S}_{B}$ can be viewed as a $k+1$ place relation.) Let $\mathcal{R}_{0}$ be the equality relation over $\mathcal{U}_{B},\left\{\langle x, x\rangle \mid x \in \mathcal{U}_{B}\right\}$. An encoding of $\mathcal{S}_{B}$ in $\mathcal{S}_{A}$ is a $k$-place relation $\mathcal{T} \subset \mathcal{U}_{A}^{k}$ and a surjection $\Gamma$ from $\mathcal{T}$ onto $\mathcal{U}_{B}$ such that for $i=0 \ldots q$, the inverse image $\Gamma^{-1}\left(\mathcal{R}_{i}\right)$ is first-order definable in $\mathcal{S}_{A}$. (Note that $\mathcal{T}$ itself is the projection of the inverse image of the equality relation over $\mathcal{U}_{B}$, and thus first-order definable in $\mathcal{S}_{A}$.)

Let $\beta\left(x_{1} \ldots x_{q}\right)$ be a first-order formula over $\mathcal{S}_{B}$, where $x_{1} \ldots x_{q}$ are open in $\beta$. It is easily seen that $\beta$ can be translated into a formula $\alpha$ over $\mathcal{S}_{A}$ as follows: replace every variable $x_{i}$ in $\beta$ by the $k$-variables $y_{i, 1} \ldots y_{i, k}$, restricted by the formula in $\mathcal{L}_{A}$ that defines $\mathcal{T}$, and replace every non-logical symbol or equality sign in $\beta$ by the formula that defines it in $\mathcal{L}_{A}$. Then $c_{1,1} \ldots c_{q, k} \in \mathcal{U}_{A}$ satisfy $\alpha$ if and only if $\Gamma\left(c_{1,1} \ldots c_{1, k}\right) \ldots \Gamma\left(c_{q, 1} \ldots c_{q, k}\right)$ satisfy $\beta$. In particular, for $q=0, \alpha$ is true if and only if $\beta$ is true.

\footnotetext{
${ }^{1}$ This is somewhat less general than the definition in Hodges [13] p. 248, but it is simpler and it is sufficient for our purposes here.
} 
Structure $\mathcal{S}_{B}$ is (first-order) definable in $\mathcal{S}_{A}$ if there exists an encoding of $\mathcal{S}_{B}$ in $\mathcal{S}_{A}$. Structures $\mathcal{S}_{A}$ and $\mathcal{S}_{B}$ are mutually definable if each is definable in the other. It is immediate that first-order definability is transitive, and hence mutual definability is an equivalence relation.

A minor discrepancy between the above definition of "definability" and the actual encodings that we will use in this paper should be noted. Definition 1 assumes that every element of $\mathcal{U}_{B}$ is encoded by a $k$-tuple of elements of $\mathcal{U}_{A}$ where $k$ is fixed, but our encodings will encode different kinds of elements with tuples of different lengths. The point is somewhat important, because we will be constructing encodings in which $\mathcal{U}_{A}$ is a proper subset of $\mathcal{U}_{B}$, and in which the encoding is the identity over $\mathcal{U}_{A}$, which requires that $k=1$ on elements of $\mathcal{U}_{A}$ and $k>1$ on elements of $\mathcal{U}_{B}-\mathcal{U}_{A}$. The discrepancy can be easily fixed in either of two ways. The more principled, but more complicated, solution, is to define $\mathcal{S}_{B}$ as a sorted structure, and allow different values of $k$ on different sorts. The kludgier but simpler solution is to "pad" out the encodings that are too short so as to achieve a uniform length, and replace the condition that the function $\Gamma$ is the identify over elements $\mathcal{U}_{B}$ with the condition that it is a projection onto the first element of the encoding tuple. It makes no difference to the eventual theorems.

The class of analytical relations can be defined as follows: (This definition is adapted from Hinman [12]; see especially section 3.5, pp. 114-124).

Definition 2: Let $\omega$ be the set of natural numbers and let $\omega^{\omega}$ be the set of infinite sequences of natural numbers (total functions from $\omega$ to $\omega$.) For a sequence $\hat{s}$ and number $p$ let $\hat{s}[p]$ be the $p$ th element of $\hat{s}$; thus [] is the two-place indexing function. Consider the structure $\mathcal{S}=\left\langle\omega \cup \omega^{\omega},+, \cdot,[]\right\rangle$. A relation is analytical if it is first-order definable over $\mathcal{S}$. The class of analytical relations is denoted $\Delta_{\omega}^{1}$.

By Gödel's theorem, any computable function can be expressed in the language over the natural numbers with + and $\cdot$; hence the class of analytical relations includes all computable functions. Likewise, it includes any function mapping sequences $\hat{s}_{1} \ldots \hat{s}_{m}$ to a sequence $\hat{t}$ if each element of $\hat{t}$ is a computable function of initial subsequences of the $\hat{s}_{i}$. (This is a sufficient condition, but by no means a necessary one.)

Definition 3: A structure $\mathcal{S}$ is complete for the class of analytical relations if it is mutually definable with the structure $\left\langle\omega \cup \omega^{\omega},+, \cdot,[]\right\rangle$

Lemma 1: Let $\mathcal{B} \subset\{0,1\}^{\omega}$ be the set of all infinite sequences of bits 0 and 1 with infinitely many 0 's. The structure $\langle\omega \cup \mathcal{B},+, \cdot,[]\rangle$ is mutually definable with $\left\langle\omega \cup \omega^{\omega},+, \cdot,[]\right\rangle$

Proof: It is trivial that $\langle\mathcal{B},+, \cdot,[]\rangle$ is definable in $\left\langle\omega \cup \omega^{\omega},+, \cdot,[]\right\rangle$ under the identity mapping. To show the converse, we encode the sequence of natural numbers $n_{1}, n_{2} \ldots$ as the bit string containing $n_{1} 1$ 's followed by 0 followed by $n_{2} 1$ 's followed by 0 , and so on. It is easily seen that the recovery of the original sequence from the bit string is definable in $\langle\omega \cup \mathcal{B},+, \cdot,[]\rangle$. I

Lemma 2: Let $\mathcal{B}^{\omega}$ be the set of all sequences of bitstrings. Extend the indexing function $\hat{s}[p]$ to map a sequence of bitstrings $s$ and an integer index $p$ to the $p$ th bitstring in $s$. Then the structure $\left\langle\omega \cup \mathcal{B} \cup \mathcal{B}^{\omega},+, \cdot,[]\right\rangle$ is mutually definable with $\langle\omega \cup \mathcal{B},+, \cdot,[]\rangle$

Proof: Use dovetailing to encode the sequence of sequences $\hat{s}_{0}, \hat{s}_{1} \ldots$ as the single sequence $\hat{s}_{0}[0], \hat{s}_{0}[1], \hat{s}_{1}[0], \hat{s}_{0}[2], \hat{s}_{1}[1], \hat{s}_{2}[0], \ldots$. The indexing function from a sequence of sequences, thus encoded, to a sequence is defined in $\langle\omega \cup \mathcal{B},+, \cdot,[]\rangle$ as follows.

Index $2(\hat{s}, i, \hat{t}): \hat{s}[i]=t$ where $s$ is the encoding of a sequence of sequences.

(2.1) Index $2(\hat{s}, i, \hat{t}) \equiv \forall_{j} \hat{t}[j]=\hat{s}\left[\left((i+j+1)^{2}+(i-j)-1\right) / 2\right]$

I

Lemma 3: Let "Integer $(\bar{x})$ " be the relation over $\Re, ~ " \bar{x}$ is an integer." The structure $\langle\Re,+, \cdot$, Integer $\rangle$ 
is mutually definable with $\langle\omega \cup \mathcal{B},+, \cdot,[]\rangle$.

Proof: To define $\langle\omega \cup \mathcal{B},+, \cdot,[])\rangle$ in $\langle\Re,+, \cdot$, Integer $\rangle$, we encode an integer as the corresponding real number, and a bit string $b_{1}, b_{2} \ldots$ as the real number $0 . b_{1} b_{2} \ldots$ in base 2 . (Note that our requirement that the bit string contain infinitely many 0 's means that we do not have to worry about $0.011111 \ldots$ being the same real number as $0.1000 \ldots$...) Integer plus and times map into real plus and times. The extraction of the $k$ th bit in the sequence corresponds to extraction of the $k$ th bit in the real number:

Floor $(\bar{x}, p): p=\lfloor\bar{x}\rfloor$.

(2.2) Floor $(\bar{x}, p) \equiv \operatorname{Integer}(p) \wedge p \leq \bar{x} \wedge \forall_{q}[\operatorname{Integer}(q) \wedge q \leq \bar{x}] \Rightarrow q \leq p$.

$\operatorname{KthBit}(\bar{x}, k, b)$ : The $k$ th bit of $x$ is $b$.

(2.3) $\operatorname{KthBit}(\bar{x}, k, b) \equiv \operatorname{Floor}\left(\bar{x} \cdot 2^{k}, p\right) \wedge b=p \bmod 2$.

Since 2, exponentiation, and mod are computable, they are definable in the language of plus and times over the natural numbers.

To define $\langle\Re,+, \cdot$, Integer $\rangle$, in $\langle\omega \cup \mathcal{B},+, \cdot,[]\rangle$, we encode real number $\bar{r}$ as a triple $\langle s, i, \hat{b}\rangle$ where the sign $s$ is 0 if $\bar{r} \geq 0$ and 1 otherwise; the integer part $i=\lfloor|\bar{r}|\rfloor$; and the bit-string $\hat{b}$ is the binary representation of $|\bar{r}|-i$. To demonstrate the encoding, we will show how the sum of two positive real numbers is defined in a formula over the corresponding bitstrings; the other definitions are analogous.

$\operatorname{PosLess}(a, b, \hat{c} ; d, e, \hat{f})$ : The real $\langle a, b, \hat{c}\rangle$ is positive and less than the real $\langle d, e, \hat{f}\rangle$.

(2.4) PosLess $(a, b, \hat{c} ; d, e, \hat{f}) \equiv$

$$
\begin{aligned}
& a=0 \wedge d=0 \wedge \\
& {[b<e \vee} \\
& {\left[b=e \wedge \exists_{q} \hat{c}[q]=0 \wedge \hat{f}[q]=1 \wedge \forall_{p} p<q \Rightarrow \hat{c}[p]=\hat{f}[p]\right]}
\end{aligned}
$$

$\operatorname{PosLeq}(a, b, \hat{c} ; d, e, \hat{f})$ : The real $\langle a, b, \hat{c}\rangle$ is positive and less than or equal to the real $\langle d, e, \hat{f}\rangle$.

(2.5) $\operatorname{PosLeq}(a, b, \hat{c} ; d, e, \hat{f}) \equiv \neg \operatorname{PosLess}(d, e, \hat{f} ; a, b, \hat{c})$.

$\operatorname{Bits2Int}(a, b, \hat{c}, k ; p): p=\left\lfloor\bar{r} \cdot 2^{k}\right\rfloor$ where $\bar{r} \geq 0$ is encoded as $\langle a, b, c\rangle$.

(2.6) $\operatorname{Bits} 2 \operatorname{Int}(a, b, \hat{c}, k ; p) \equiv a=0 \wedge p=b \cdot 2^{k}+\sum_{i=1}^{k} \hat{c}[i] \cdot 2^{k-i}$.

Since the right-hand side of the above equation is computable using only $k$ bits of $\bar{r}$, it is definable in $\langle\omega \cup \mathcal{B},+, \cdot,[]\rangle$.

PlusLB/PlusUB $(a, b, \hat{c} ; d, e, \hat{f} ; g, h, i ; \hat{k})$ : Let real numbers $\bar{x}, \bar{y}, \bar{z}$ be represented by $\langle a, b, \hat{c}\rangle$, $\langle d, e, \hat{f}\rangle,\langle g, h, \hat{i}\rangle$, respectively. Then $\bar{z}$ is a lower/upper bound for $\bar{x}+\bar{y}$ as determined by a calculation to $k$ bits.

$$
\begin{aligned}
& \text { (2.7) } \operatorname{PlusLB}(a, b, \hat{c} ; d, e, \hat{f} ; g, h, \hat{i} ; k) \equiv \\
& \quad \exists_{p, q} \operatorname{Bits} 2 \operatorname{Int}(a, b, \hat{c}, k ; p) \wedge \operatorname{Bits} 2 \operatorname{Int}(d, e, \hat{f}, k ; q) \wedge \operatorname{Bits} 2 \operatorname{Int}(g, h, \hat{i}, k ; p+q) . \\
& \text { (2.8) PlusUB }(a, b, \hat{c} ; d, e, \hat{f} ; g, h, \hat{i} ; k) \equiv \\
& \quad \exists_{p, q} \operatorname{Bits} 2 \operatorname{Int}(a, b, \hat{c}, k ; p) \wedge \operatorname{Bits} 2 \operatorname{Int}(d, e, \hat{f}, k ; q) \wedge \operatorname{Bits} 2 \operatorname{Int}(g, h, \hat{i}, k ; p+q+2) .
\end{aligned}
$$

$\operatorname{PosPlus}(a, b, \hat{c} ; d, e, \hat{f} ; g, h, \hat{i})$ : Let positive real numbers $\bar{x}, \bar{y}, \bar{z}$ be represented by $\langle a, b, \hat{c}\rangle$, $\langle d, e, \hat{f}\rangle,\langle g, h, \hat{i}\rangle$, respectively. Then $\bar{z}=\bar{x}+\bar{y}$.

(2.9) PosPlus $(a, b, \hat{c} ; d, e, \hat{f} ; g, h, \hat{i}) \equiv$

$$
\begin{aligned}
& \forall_{k} \exists_{m, n, \hat{p}, q, r, \hat{s}} \\
& \quad \operatorname{PlusUB}(a, b, \hat{c} ; d, e, \hat{f} ; m, n, \hat{p} ; k) \wedge \operatorname{PlusUB}(a, b, \hat{c} ; d, e, \hat{f} ; q, r, \hat{s} ; k) \wedge \\
& \quad \operatorname{PosLeq}(m, n, \hat{p} ; g, h, \hat{i}) \wedge \operatorname{PosLeq}(g, h, \hat{i} ; q, r, \hat{s})
\end{aligned}
$$


Lemma 4: Let $\Re^{\omega}$ be the set of infinite sequences of real numbers. Define the function $\hat{s}[p]$ to be the $p$ th element if $s$ is a sequence and $p$ is an integer and 0 otherwise. Then the structure $\left\langle\Re \cup \Re^{\omega},+, \cdot,[]\right.$, Integer $\rangle$ can be defined in the structure $\langle\Re,+, \cdot$, Integer $\rangle$.

Proof: A sequence of real numbers $\bar{x}_{0}, \bar{x}_{1} \ldots$ is encoded by:

- Using lemma 3 , encode each $\bar{x}_{i}$ as a triple $a_{i}, b_{i}, \hat{c}_{i}$.

- Using lemma 3 , encode the bit string $a_{0}, a_{1} \ldots$ (with associated sign and integer part equal to $0)$ as a real number.

- Using lemma 1 , encode the integer sequence $b_{0}, b_{1} \ldots$ as a bit string. Using Lemma 3 , encode this bit string as a real number.

- Using lemma 2 , dovetail the bit strings $\hat{c}_{0}, \hat{c}_{1} \ldots$ into a single bit string. Using lemma 3 , encode this bit string as a real number.

Thus we have encoded an infinite sequence of real numbers as three real numbers. Defining the indexing function $\hat{s}[p]$ from a sequence of reals $\hat{s}$ encoded as a triple $\langle a, b, \hat{c}\rangle$ and an integer $p$ to the real $\hat{s}[p]$ is just a question of undoing this encoding using the predicates that we have defined. Note that the predicate for translating a bitstring into a sequence of natural numbers, defined in $\left\langle\omega \cup \omega^{\omega},+, \cdot,[]\right\rangle$ and the dovetailing predicate "Index2" must be translated into $\langle\Re,+, \cdot$, Integer $\rangle$, but we have shown that that can be done.

A trivial corollary is that it is possible to encode sequences of pairs of real numbers; the sequence $\langle\bar{x}[0], \bar{y}[0]\rangle,\langle\bar{x}[1], \bar{y}[1]\rangle \ldots$ can be encoded as the sequence $\bar{x}[0], \bar{y}[0], \bar{x}[1], \bar{y}[1] \ldots$

PairIndex $(\hat{s}, k, \bar{x}, \bar{y})$ - The pair $\langle\bar{x}, \bar{y}\rangle$ is the $k$ th pair in the sequence $\hat{s}$ of real numbers.

(2.10) PairIndex $(\hat{s}, k, \bar{x}, \bar{y}) \equiv \bar{x}=\hat{s}[k+k] \wedge \bar{y}=\hat{s}[k+k+1]$.

\section{The Language of Connection and Convexity}

We now turn to the main point of this paper; establishing the expressivity of a few small first-order languages over a universe of spatial regions. We begin with the structure containing the two relations "Convex $(x)$ " (region $x$ is convex) and " $\mathrm{C}(x, y)$ " (regions $x$ and $y$ have at least one point in common). To simplify the exposition, in this section and section 4 we will develop our theory over regions in the two-dimensional plane, but it is straightforward to extend the results to higher-dimensional Euclidean space.

In this section and the next, let $\mathcal{U}$ be a collection of non-empty closed regions in the plane, such that $\mathcal{U}$ contains every solid simple polygon. A region is regular if it is non-empty and equal to the closure of its interior. The boundary of region $R$ is the difference $\operatorname{Closure}(R)-\operatorname{Interior}(R)$. A polygon is a bounded, regular region whose boundary is the union of a finite set of line segments. A polygon is simple if it is homeomorphic to a closed disk (equivalently its boundary is a simple closed curve).

We emphasize that the only constraints on $\mathcal{U}$ are that all the regions in $\mathcal{U}$ are closed and that all simple solid polygons are in $\mathcal{U}$. For all closed regions that are not simple solid polygons - curved regions, regions with holes, disconnected regions, pathological regions - you can choose to include or exclude any that you wish. The object of this level of generality is so that the theorem will cover just about any desired notion of what is a "well-behaved" region. However, it does mean that one 


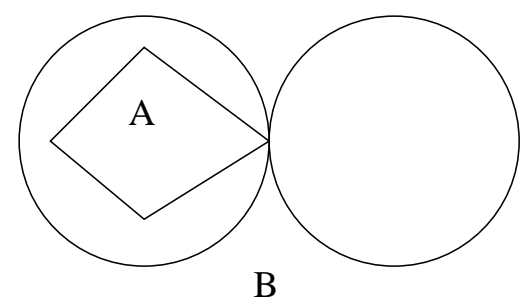

$\mathrm{A}$ is the quadrilateral; B is the union of the two disks.

Any polygon connected to A overlaps B.

Figure 1: An anomaly in a strange universe of regions.

has to be more careful with definitions and rely less on intuition than usual. For example, in many spatial languages over regions, one can assume that if $r$ is a region, then there exist regions that are proper subsets of $r$. But that may not be true in our universe; we can, for example, define $\mathcal{U}$ to be the set of all solid polygons union the set of all lines of length 1 . Another example: in most standard universes, if $a \subset b$ and $a$ meets the boundary of $b(\operatorname{TPP}(a, b))$ then $a$ is connected to some region outside $b$. But consider the universe $\mathcal{U}$ consisting of all polygons together with a region $b$ that is the union of two circles that are tangent at the origin. Let $a \subset b$ be a region that meets the boundary of $b$ just at the origin. Then there is no region $c \in \mathcal{U}$ that is outside $b$ and meets $a$, because no polygon can squeeze its way into the cusp of the complement of $b$ at the origin (Figure 1).

(Note that just proving Theorem 1 over the smallest universe - the set of all simple solid polynomials - would not suffice to prove the more general result, because a structure with a larger universe may be less expressive. For example, the theory with addition and multiplication is undecidable over the integers but decidable over the reals.)

We begin by defining some basic topological relations:

$\mathrm{P}(x, y)$ : Region $x$ is a subset of region $y$.

(3.1) $\mathrm{P}(x, y) \equiv \forall_{z} \mathrm{C}(z, x) \Rightarrow \mathrm{C}(z, y)$.

$\mathrm{PP}(x, y)$ : Region $x$ is a proper subset of region $y$.

(3.2) $\mathrm{PP}(x, y) \equiv \mathrm{P}(x, y) \wedge \neg \mathrm{P}(y, x)$.

BetweenCH $(a, b, c)$ : Region $b$ lies in the convex hull of $a \cup c$.

(3.3) $\operatorname{BetweenCH}(a, b, c) \equiv$

$$
\forall_{x}[\operatorname{Convex}(x) \wedge \mathrm{P}(a, x) \wedge \mathrm{P}(c, x)] \Rightarrow \mathrm{P}(b, x) .
$$

ConvexSolid $(x): x$ is a convex region with non-empty interior (i.e. not a single point or line segment) if it contains $a, b, c$ none of which is between the other.

(3.4) $\operatorname{ConvexSolid}(x) \equiv$

$$
\begin{aligned}
& \text { Convex }(x) \wedge \\
& \exists_{a, b, c} \mathrm{P}(a, x) \wedge \mathrm{P}(b, x) \wedge \mathrm{P}(c, x) \wedge \neg \mathrm{C}(a, b) \wedge \neg \mathrm{C}(b, c) \wedge \neg \mathrm{C}(a, c) \wedge \\
& \neg \operatorname{BetweenCH}(a, b, c) \wedge \neg \operatorname{BetweenCH}(b, c, a) \wedge \neg \operatorname{BetweenCH}(c, a, b) .
\end{aligned}
$$

Note that definition 3.4 is correct if $a, b, c$ are restricted to being polygons, or if they are allowed to be arbitrary point sets, or anything in between. Note also that every region satisfying this predicate is regular.

(3.5) Union $(p, q, r): r=p \cup q$.

$\operatorname{Union}(p, q, r) \equiv \forall_{x} \mathrm{C}(x, r) \Leftrightarrow \mathrm{C}(x, p) \vee \mathrm{C}(x, q)$.

Conn $(r)$ : Region $r$ is connected.

(3.6) $\operatorname{Conn}(r) \equiv \forall_{p, q} \operatorname{Union}(p, q, r) \Rightarrow \mathrm{C}(p, q)$ 
$\mathrm{O}(x, y): x$ and $y$ overlap; that is, $x \cap y$ has a non-empty interior.

(3.7) $\mathrm{O}(x, y) \equiv \exists_{z}$ ConvexSolid $(z) \wedge \mathrm{P}(z, x) \wedge \mathrm{P}(z, y)$.

$\mathrm{EC}(r, s)$ : Regions $r$ and $s$ are externally connected.

(3.8) $\mathrm{EC}(r, s) \equiv \mathrm{C}(r, s) \wedge \neg \mathrm{O}(r, s)$.

We next show that a point $\mathbf{p}$ can be encoded in terms of two convex solids $a, b$ that meet only at $\mathbf{p}$.

Lemma 5: If $a, b$ are convex solids and points $\mathbf{p} \neq \mathbf{q}$ are both in $a \cap b$, then there exist polygons $c, d$ such that $\mathbf{p} \in c \subset a, \mathbf{q} \in d \subset b$, and $c \cap d=\emptyset$.

The proof is immediate. Note that the reason we require that $c$ and $d$ be polygons is that these are the only kinds of regions that are required to be in $\mathcal{U}$, and the reason $a$ and $b$ are required to be convex solids is that the consequence is not true without that.

Lemma 6 (Adapted from Schaefer and Stefankovic [19]): If $a, b$ are convex solids such that $\mathrm{C}(a, b)$, then $a$ and $b$ meet in a single point if and only if the following holds: for all regions $c, d$ in $\mathcal{U}$, if $c \subset a, d \subset b, \mathrm{C}(c, b)$, and $\mathrm{C}(d, a)$ then $\mathrm{C}(c, d)$.

Proof: The implication left to right is trivial, and the implication right to left is just the contrapositive of lemma 5 .

Using lemma 6 , we can define the property of regions $a, b$ being convex solid regions that meet only at a single point; and we can therefore use such a pair as the encoding of a point.

IsPoint $(a, b)$ : Regions $a$ and $b$ encode a point, as above.

(3.9) $\operatorname{IsPoint}(a, b) \equiv$

ConvexSolid $(a) \wedge$ ConvexSolid $(b) \wedge$

$\forall_{c, d}[\mathrm{P}(c, a) \wedge \mathrm{P}(d, b) \wedge \mathrm{C}(c, b) \wedge \mathrm{C}(d, a)] \Rightarrow \mathrm{C}(c, d)$.

We now complete the encoding by showing how the identity relation on points and basic relations between points and regions can be defined: Let $a, b$ meet at the single point $\mathbf{p}$ and let $c, d$ meet at the single point $\mathbf{q}$. Then $\mathbf{p}=\mathbf{q}$ if and only if the following holds: For every regions $w, x, y, z$, if $w \subset a, x \subset b, y \subset c, z \subset d, \mathrm{C}(w, x)$, and $\mathrm{C}(y, z)$ then $\mathrm{C}(w, y)$.

SamePoint $(a, b ; c, d)$ : Point $\langle a, b\rangle$ is the same as point $\langle c, d\rangle$.

(3.10) SamePoint $(a, b ; c, d) \equiv$

$\operatorname{IsPoint}(a, b) \wedge \operatorname{IsPoint}(c, d) \wedge$

$\left[\forall_{w, x, y, z} \mathrm{P}(w, a) \wedge \mathrm{P}(x, b) \wedge \mathrm{P}(y, c) \wedge \mathrm{P}(z, d) \wedge \mathrm{C}(w, x) \wedge \mathrm{C}(y, z) \Rightarrow \mathrm{C}(w, y)\right]$.

ConvexNTPP $(a, b): a$ and $b$ are convex regions, and $a \subset \operatorname{Interior}(b)$.

(3.11) ConvexNTPP $(a, b) \equiv$

Convex $(a) \wedge$ Convex $(b) \wedge \mathrm{PP}(a, b) \wedge$

$\forall_{c}[$ ConvexSolid $(c) \wedge \mathrm{C}(c, a)] \Rightarrow \mathrm{O}(c, b)$.

$\operatorname{InPt}(a, b ; c)$ : Point $\langle a, b\rangle$ is in region $c$.

(3.12) $\operatorname{InPt}(a, b ; c) \equiv \forall_{d, e} \operatorname{SamePoint}(d, e ; a, b) \Rightarrow \mathrm{C}(d, c)$.

$\operatorname{IntPt}(a, b ; c)$ : Point $\langle a, b\rangle \in \operatorname{Interior}(c)$.

(3.13) $\operatorname{IntPoint}(a, b ; c) \equiv$

$\exists_{d, e, f, g} \operatorname{SamePoint}(d, e ; a, b) \wedge \mathrm{P}(d, f) \wedge \mathrm{P}(e, f) \wedge \operatorname{ConvexNTPP}(f, g) \wedge \mathrm{P}(g, c)$.

$\operatorname{BdPt}(a, b ; c):$ Point $\langle a, b\rangle \in \operatorname{Boundary}(c)$.

(3.14) $\operatorname{BdPt}(a, b, c) \equiv \operatorname{InPt}(a, b ; c) \wedge \neg \operatorname{IntPt}(a, b ; c)$.

Having defined points, it will simplify the exposition if we directly include them in the structure, rather than always having to refer to them as pairs of convex solids. Let $\mathcal{P}$ be the class of points. 
We extend the universe under consideration to be $\mathcal{U} \cup \mathcal{P}$ and add the relations InPt, and BdPt; and and we note that we have proven that:

Lemma 7: The stucture $\langle\mathcal{U} \cup \mathcal{P}$, C,Convex,InPt,BdPt $\rangle$ is definable in $\langle\mathcal{U}$, C,Convex $\rangle$ under a encoding which is the identity over all of $\mathcal{U}$.

Continuing on in this new enriched language, we now turn to relations in affine geometry. Here we are following [16] and [6] though with some differences; [16] includes half-planes as regions, which simplified some of the definitions, and [6] works in a constraint language, which is substantially less expressive, and therefore requires more convoluted definitions. We will use bold-face letters to indicate variables with sort "point".

Collinear $(\mathbf{p}, \mathbf{q}, \mathbf{r})$ : Points $\mathbf{p}, \mathbf{q}, \mathbf{r}$ are collinear if they lie on the boundaries of regions $a$ and $b$ which are convex and EC.

(3.15) Collinear $(\mathbf{p}, \mathbf{q}, \mathbf{r}) \equiv$

$$
\begin{aligned}
& \exists_{a, b} \operatorname{EC}(a, b) \wedge \operatorname{Convex}(a) \wedge \operatorname{Convex}(b) \wedge \\
& \operatorname{BdPt}(\mathbf{p}, a) \wedge \operatorname{BdPt}(\mathbf{p}, b) \wedge \operatorname{BdPt}(\mathbf{q}, a) \wedge \operatorname{BdPt}(\mathbf{q}, b) \wedge \operatorname{BdPt}(\mathbf{r}, a) \wedge \operatorname{BdPt}(\mathbf{r}, b) .
\end{aligned}
$$

$\operatorname{Order}(\mathbf{p}, \mathbf{q}, \mathbf{r})$ : Point $\mathbf{q}$ lies on the line from $\mathbf{p}$ to $\mathbf{r}$, strictly between them.

(3.16) Order $(\mathbf{p}, \mathbf{q}, \mathbf{r}) \equiv$

$$
\begin{aligned}
& \text { Collinear }(\mathbf{p}, \mathbf{q}, \mathbf{r}) \wedge \\
& \exists_{a} \text { Convex }(a) \wedge \operatorname{InPt}(\mathbf{p}, a) \wedge \operatorname{InPt}(\mathbf{q}, a) \wedge \neg \operatorname{InPt}(\mathbf{r}, a) \wedge \\
& \exists_{b} \text { Convex }(b) \wedge \operatorname{InPt}(\mathbf{r}, b) \wedge \operatorname{InPt}(\mathbf{q}, b) \wedge \neg \operatorname{InPt}(\mathbf{p}, b) .
\end{aligned}
$$

$\operatorname{Parallel}(\mathbf{p}, \mathbf{q}, \mathbf{r}, \mathbf{s})$ : The line from $\mathbf{p}$ to $\mathbf{q}$ is parallel (and unequal) to the line from $\mathbf{r}$ to $\mathbf{s}$.

(3.17) Parallel $(\mathbf{p}, \mathbf{q}, \mathbf{r}, \mathbf{s}) \equiv \neg \exists_{\mathbf{z}}$ Collinear $(\mathbf{z}, \mathbf{p}, \mathbf{q}) \wedge$ Collinear $(\mathbf{z}, \mathbf{r}, \mathbf{s})$

Parallelogram $(\mathbf{p}, \mathbf{q}, \mathbf{r}, \mathbf{s})$ : Points $\mathbf{p}, \mathbf{q}, \mathbf{r}, \mathbf{s}$ form a parallelogram (in that order).

(3.18) Parallelogram $(\mathbf{p}, \mathbf{q}, \mathbf{r}, \mathbf{s}) \equiv \operatorname{Parallel}(\mathbf{p}, \mathbf{q}, \mathbf{s}, \mathbf{r}) \wedge \operatorname{Parallel}(\mathbf{p}, \mathbf{s}, \mathbf{q}, \mathbf{r})$.

EqualVec $(\mathbf{p}, \mathbf{q} ; \mathbf{r}, \mathbf{s})$ : Vectors $\overrightarrow{\mathbf{p q}}=\overrightarrow{\mathbf{r s}}$.

(3.19) EqualVec $(\mathbf{p}, \mathbf{q} ; \mathbf{r}, \mathbf{s}) \equiv$

$[(\mathbf{p}=\mathbf{q}) \wedge(\mathbf{r}=\mathbf{s})] \vee$

$\exists_{\mathbf{w}, \mathbf{x}}$ Parallelogram $(\mathbf{p}, \mathbf{q}, \mathbf{w}, \mathbf{x}) \wedge$ Parallelogram $(\mathbf{w}, \mathbf{x}, \mathbf{r}, \mathbf{s})$.

Translate $(a, \mathbf{p}, \mathbf{q}, b)$ : Translating region $a$ by vector $\overrightarrow{\mathbf{p q}}$ gives region $b$.

(3.20) Translate $(a, \mathbf{p}, \mathbf{q}, b) \equiv$ $\forall_{\mathbf{x}, \mathbf{y}} \operatorname{EqualVec}(\mathbf{p}, \mathbf{q} ; \mathbf{x}, \mathbf{y}) \Rightarrow[\operatorname{InPt}(\mathbf{x}, a) \Leftrightarrow \operatorname{InPt}(\mathbf{y}, b)]$.

$\operatorname{VecPlus}(\mathbf{a}, \mathbf{b} ; \mathbf{c}, \mathbf{d} ; \mathbf{e}, \mathbf{f}): \mathbf{a \mathbf { b }}+\overrightarrow{\mathbf{c d}}=\overrightarrow{\mathbf{e f}}$.

(3.21) $\operatorname{VecPlus}(\mathbf{a}, \mathbf{b} ; \mathbf{c}, \mathbf{d} ; \mathbf{e}, \mathbf{f}) \equiv \exists_{\mathbf{g}} \operatorname{EqualVec}(\mathbf{b}, \mathbf{g} ; \mathbf{c}, \mathbf{d}) \wedge \operatorname{EqualVec}(\mathbf{a}, \mathbf{g} ; \mathbf{e}, \mathbf{f})$

$\operatorname{AfCoorSys}(\mathbf{o}, \mathbf{i}, \mathbf{j})$ Points $\mathbf{o}, \mathbf{i}, \mathbf{j}$ form an affine coordinate system if they are not collinear.

Point $\mathbf{o}$ is the origin, and $\mathbf{i}$ and $\mathbf{j}$ are the unit points on the $\mathrm{x}$ - and $\mathrm{y}$-axes.

(3.22) AfCoorSys $(\mathbf{o}, \mathbf{i}, \mathbf{j}) \equiv \neg$ Collinear $(\mathbf{o}, \mathbf{i}, \mathbf{j})$.

$\operatorname{AfCoors}(\mathbf{p}, \mathbf{x}, \mathbf{y} ; \mathbf{o}, \mathbf{i}, \mathbf{j})$ : Points $\mathbf{p}$ has coordinates $\mathbf{x}$ and $\mathbf{y}$ in coordinate systems $\mathbf{o}, \mathbf{i}, \mathbf{j}$.

(Figure 2).

(3.23) $\operatorname{AfCoors}(\mathbf{p}, \mathbf{x}, \mathbf{y} ; \mathbf{o}, \mathbf{i}, \mathbf{j}) \equiv$ $\operatorname{AfCoorSys}(\mathbf{o}, \mathbf{i}, \mathbf{j}) \wedge \operatorname{Collinear}(\mathbf{o}, \mathbf{i}, \mathbf{x}) \wedge \operatorname{Collinear}(\mathbf{o}, \mathbf{j}, \mathbf{y}) \wedge \operatorname{VecPlus}(\mathbf{o}, \mathbf{x} ; \mathbf{o}, \mathbf{y} ; \mathbf{o}, \mathbf{p})$.

$\operatorname{Ratio}(\mathbf{o}, \mathbf{i}, \mathbf{x}, \mathbf{j}, \mathbf{y}): \mathbf{o}, \mathbf{i}, \mathbf{j}$ are not collinear and $|\mathbf{o x}| /|\mathbf{o i}|=|\mathbf{o y}| /|\mathbf{o j}|$ (Figure 3).

(3.24) Ratio $(\mathbf{o}, \mathbf{i}, \mathbf{x}, \mathbf{j}, \mathbf{y}) \equiv$

$\operatorname{AfCoorSys}(\mathbf{o}, \mathbf{i}, \mathbf{j}) \wedge$

$\exists_{\mathbf{q}, \mathbf{p}} \operatorname{AfCoors}(\mathbf{p}, \mathbf{i}, \mathbf{j} ; \mathbf{o}, \mathbf{i}, \mathbf{j}) \wedge \operatorname{AfCoors}(\mathbf{q}, \mathbf{x}, \mathbf{y} ; \mathbf{o}, \mathbf{i}, \mathbf{j}) \wedge \operatorname{Collinear}(\mathbf{o}, \mathbf{p}, \mathbf{q})$.

At this point, we can define real arithmetic. A real number $\bar{r}$ is encoded as a triple of collinear points $\langle\mathbf{p} ; \mathbf{o}, \mathbf{i}\rangle$, where $\bar{r}$ is the coordinate of $\mathbf{p}$ on the $\mathbf{o}, \mathbf{i}$ axis. We use vector addition to define real 


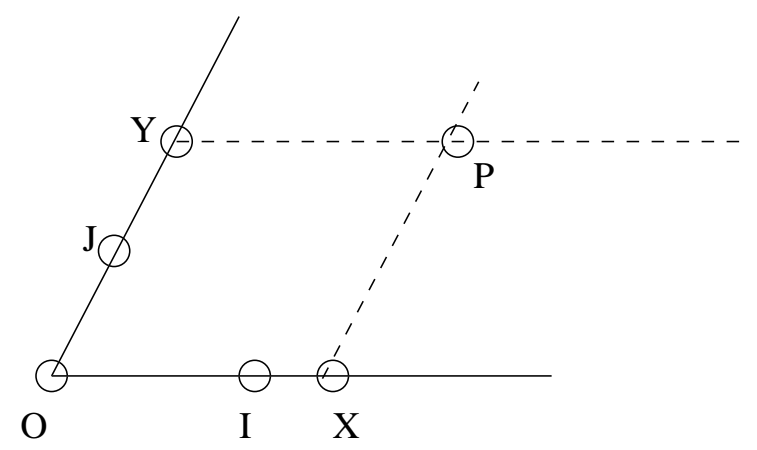

Figure 2: An affine coordinate system

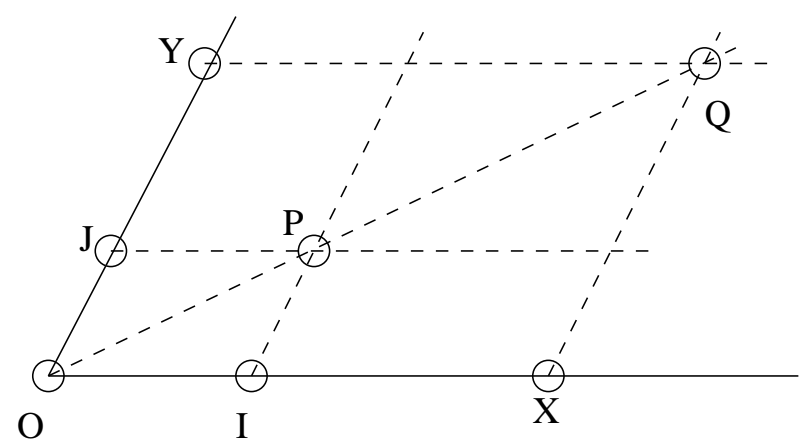

Figure 3: Ratio of lengths

addition and the "Ratio" predicate just defined both to define multiplication and to identify the same real number in two different coordinate systems.

IsReal $(\mathbf{p} ; \mathbf{o}, \mathbf{i}): \mathbf{p}$ represents a real number in axis $\mathbf{o}, \mathbf{i}$.

(3.25) IsReal $(\mathbf{p} ; \mathbf{o}, \mathbf{i}) \equiv$ Collinear $(\mathbf{p}, \mathbf{o}, \mathbf{i}) \wedge \mathbf{o} \neq \mathbf{i}$.

SameReal $(\mathbf{p} ; \mathbf{o}, \mathbf{i} ; \mathbf{q} ; \mathbf{u}, \mathbf{w}):\langle\mathbf{q}: \mathbf{u}, \mathbf{w}\rangle$ represents the same real number as $\langle\mathbf{p} ; \mathbf{o}, \mathbf{i}\rangle$.

(3.26) SameReal $(\mathbf{p} ; \mathbf{o}, \mathbf{i} ; \mathbf{q} ; \mathbf{u}, \mathbf{w}) \equiv$

$\exists_{\mathbf{j}, \mathbf{y}, \mathbf{k}, \mathbf{m}, \mathbf{z}} \operatorname{EqualVec}(\mathbf{o}, \mathbf{j} ; \mathbf{u}, \mathbf{w}) \wedge \operatorname{EqualVec}(\mathbf{o}, \mathbf{y} ; \mathbf{u}, \mathbf{q}) \wedge$

$\operatorname{Ratio}(\mathbf{o}, \mathbf{i}, \mathbf{p}, \mathbf{k}, \mathbf{m}) \wedge \operatorname{Ratio}(\mathbf{o}, \mathbf{j}, \mathbf{y}, \mathbf{k}, \mathbf{m})$.

$\operatorname{Plus}(\mathbf{p} ; \mathbf{o}, \mathbf{i} ; \mathbf{q} ; \mathbf{u}, \mathbf{j} ; ; \mathbf{r} ; \mathbf{v}, \mathbf{k}):\langle\mathbf{p} ; \mathbf{o}, \mathbf{i}\rangle+\langle\mathbf{q} ; \mathbf{u}, \mathbf{j}\rangle=\langle\mathbf{r} ; \mathbf{v}, \mathbf{k}\rangle$.

(3.27) $\operatorname{Plus}(\mathbf{p} ; \mathbf{o}, \mathbf{i} ; ; \mathbf{q} ; \mathbf{u}, \mathbf{j} ; ; \mathbf{r} ; \mathbf{v}, \mathbf{k}) \equiv$

$\exists_{\mathbf{x}, \mathbf{y}} \operatorname{SameReal}(\mathbf{x} ; \mathbf{o}, \mathbf{i} ; ; \mathbf{q} ; \mathbf{u}, \mathbf{j}) \wedge \operatorname{SameReal}(\mathbf{y} ; \mathbf{o}, \mathbf{i} ; ; \mathbf{r}, \mathbf{v}, \mathbf{k}) \wedge \operatorname{Vec} \operatorname{Plus}(\mathbf{o}, \mathbf{p} ; \mathbf{o}, \mathbf{x} ; \mathbf{o}, \mathbf{y})$.

$\operatorname{Times}(\mathbf{p} ; \mathbf{o}, \mathbf{i} ; ; \mathbf{q} ; \mathbf{u}, \mathbf{j} ; ; \mathbf{r} ; \mathbf{v}, \mathbf{k}):\langle\mathbf{p} ; \mathbf{o}, \mathbf{i}\rangle \cdot\langle\mathbf{q} ; \mathbf{u}, \mathbf{j}\rangle=\langle\mathbf{r} ; \mathbf{v}, \mathbf{k}$.

(3.28) Times $(\mathbf{p} ; \mathbf{o}, \mathbf{i} ; ; \mathbf{q} ; \mathbf{u}, \mathbf{j} ; ; \mathbf{r} ; \mathbf{v}, \mathbf{k}) \equiv$

$\exists_{\mathbf{x}, \mathbf{y}} \operatorname{SameReal}(\mathbf{x} ; \mathbf{o}, \mathbf{i} ; ; \mathbf{q} ; \mathbf{u}, \mathbf{j}) \wedge \operatorname{SameReal}(\mathbf{y} ; \mathbf{o}, \mathbf{i} ; ; \mathbf{r}, \mathbf{v}, \mathbf{k}) \wedge$

$[\mathbf{x}=\mathbf{o}) \wedge \mathbf{y}=\mathbf{o})] \vee \operatorname{SameReal}(\mathbf{p} ; \mathbf{o}, \mathbf{i} ; ; \mathbf{y} ; \mathbf{o}, \mathbf{x})$.

(3.29) $\operatorname{NonNeg}(\mathbf{p} ; \mathbf{o}, \mathbf{i}) \equiv \operatorname{Order}(\mathbf{o}, \mathbf{i}, \mathbf{p}) \vee \mathbf{p}=\mathbf{i}) \vee \operatorname{Order}(\mathbf{o}, \mathbf{p}, \mathbf{i})$.

(3.30) $\operatorname{Leq}(\mathbf{p} ; \mathbf{o}, \mathbf{i} ; \mathbf{q} ; \mathbf{u}, \mathbf{j}) \equiv \exists_{\mathbf{r}} \operatorname{NonNeg}(\mathbf{r} ; \mathbf{o}, \mathbf{i}) \wedge \operatorname{Plus}(\mathbf{p} ; \mathbf{o}, \mathbf{i} ; ; \mathbf{r} ; \mathbf{o}, \mathbf{i} ; ; \mathbf{q} ; \mathbf{u}, \mathbf{j})$.

We can now add real numbers to our universe and add to our language the functions $\bar{x}+\bar{y}$, $\bar{x} \cdot \bar{y}$; and the predicates $\bar{x} \leq \bar{y}$, and $\operatorname{Coors}(\mathbf{p}, \bar{x}, \bar{y} ; \mathbf{o}, \mathbf{i}, \mathbf{j}$ ) (The coordinates of $\mathbf{p}$ in frame $\mathbf{o}, \mathbf{i}, \mathbf{j}$ are $\bar{x}, \bar{y})$. We will assume other standard numeric constants and functions $(0,1$, minus, absolute value, etc.) when we need them. 


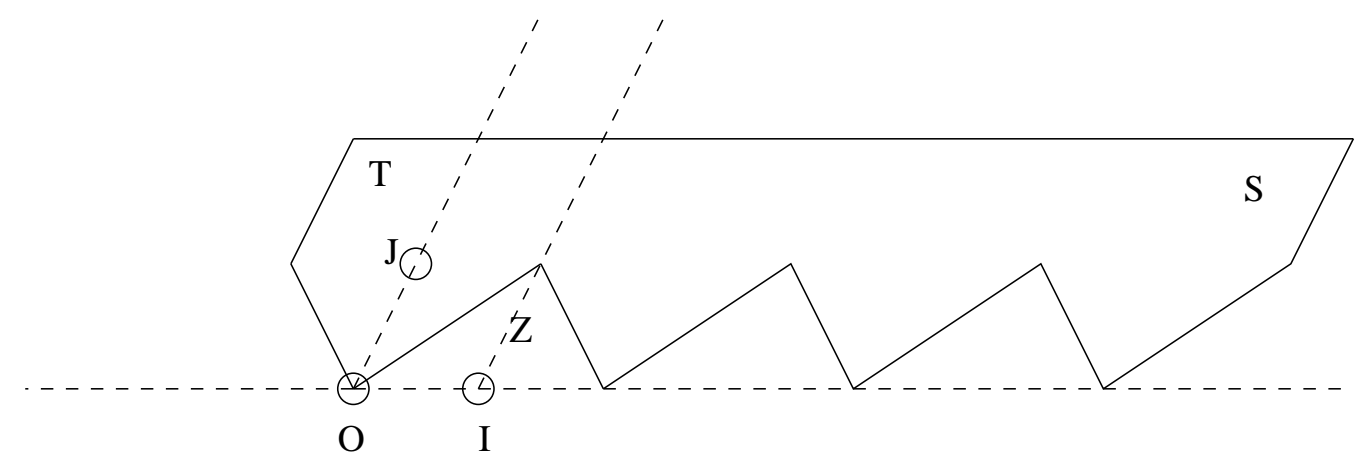

Figure 4: Sawtooth region

Finally, to represent an integer, we use a sawtooth region with regular teeth. (Figure 4).

$\operatorname{Tooth}(t ; \mathbf{o}, \mathbf{i}, \mathbf{j})$ : Region $t$ is a single tooth in coordinate system $\mathbf{o}, \mathbf{i}, \mathbf{j}$.

(3.31) $\operatorname{Tooth}(t ; \mathbf{o}, \mathbf{i}, \mathbf{j}) \equiv$

$\forall \mathbf{p} \operatorname{InPt}(\mathbf{p}, r) \Leftrightarrow$

$\exists_{\bar{x}, \bar{y}} \operatorname{AfCoors}(\mathbf{p}, \bar{x}, \bar{y} ; \mathbf{o}, \mathbf{i}, \mathbf{j}) \wedge$

$0 \leq \bar{x}+1 \wedge \bar{x} \leq 1 \wedge \bar{y} \leq 2 \wedge 0 \leq \bar{x}+\bar{y} \wedge \bar{x} \leq \bar{y}$

LowerTriangle $(z ; \mathbf{o}, \mathbf{i}, \mathbf{j}): z$ is the triangle below the tooth.

(3.32) LowerTriangle $(z ; \mathbf{o}, \mathbf{i}, \mathbf{j}) \equiv$

$\forall \mathbf{p} \operatorname{InPt}(\mathbf{p}, z) \Leftrightarrow$

$\exists_{\bar{x}, \bar{y}} \operatorname{AfCoors}(\mathbf{p}, \bar{x}, \bar{y} ; \mathbf{o}, \mathbf{i}, \mathbf{j}) \wedge 0 \leq \bar{y} \wedge \bar{y} \leq \bar{x} \wedge \bar{x}+\bar{y} \leq 2$.

$\operatorname{Saw} \operatorname{Tooth}(s ; \mathbf{o}, \mathbf{i}, \mathbf{j})-s$ is a sawtooth region w.r.t $\mathbf{o}, \mathbf{i}, \mathbf{j}$ if (a) $s$ is connected; (b) every point in $s$ is in a piece of $s$ that is a translation of the tooth in the direction; and (c) every such tooth-shaped piece of $s$ borders translations of the lower triangle that are not in $s$.

(3.33) $\operatorname{SawTooth}(s, \mathbf{o}, \mathbf{i}, \mathbf{j}) \equiv$

$$
\begin{aligned}
& \operatorname{Conn}(s) \wedge \\
& \forall \mathbf{p} \operatorname{InPt}(\mathbf{p}, s) \Rightarrow
\end{aligned}
$$

$\exists_{t, z, u, v, w, \mathbf{a}, \mathbf{b}} \operatorname{Tooth}(t ; \mathbf{o}, \mathbf{i}, \mathbf{j}) \wedge \operatorname{LowerTriangle}(z ; \mathbf{o}, \mathbf{i}, \mathbf{j}) \wedge$

Collinear $(\mathbf{o}, \mathbf{i}, \mathbf{a}) \wedge \operatorname{Translation}(t, \mathbf{o}, \mathbf{a}, u) \wedge \mathrm{P}(u, s) \wedge \operatorname{In}(\mathbf{p}, \mathrm{u}) \wedge$

$\operatorname{Translation}(z, \mathbf{o}, \mathbf{a}, v) \wedge \operatorname{EC}(v, s) \wedge$

$\operatorname{Plus}(\mathbf{b} ; \mathbf{o}, \mathbf{i} ; ; \mathbf{i} ; \mathbf{o}, \mathbf{i} ; ; \mathbf{a} ; \mathbf{o}, \mathbf{i}) \wedge \operatorname{Translation}(z, \mathbf{o}, \mathbf{b}, w) \wedge \mathrm{EC}(w, s)$.

$\operatorname{Even}(\bar{r})$ : The even numbers are where the sawtooth meets the $\mathrm{x}$-axis.

(3.34) $\operatorname{Even}(\bar{r}) \equiv$

$$
\exists_{s, \mathbf{o}, \mathbf{i}, \mathbf{j}, \mathbf{p}} \operatorname{Sawtooth}(s, \mathbf{o}, \mathbf{i}, \mathbf{j}) \wedge \operatorname{InPt}(\mathbf{p}, s) \wedge \operatorname{AfCoor}(\mathbf{p}, \bar{r}, 0 ; \mathbf{o}, \mathbf{i}, \mathbf{j}) .
$$

$\operatorname{Natural}(\bar{r})$ : The natural numbers are the non-negative evens divided by two.

(3.35) $\operatorname{Natural}(\bar{r}) \equiv \exists_{\bar{e}} \operatorname{Even}(\bar{e}) \wedge 2 \cdot \bar{r}=\bar{e} \wedge 0 \leq \bar{r}$.

(3.36) Integer $(\bar{r}) \equiv \exists_{\bar{s}} \operatorname{Natural}(\bar{s}) \wedge[\bar{r}=\bar{s} \vee \bar{r}=-\bar{s}]$.

Putting all this together with lemma 4, which states that an infinite sequence of reals and the indexing function can be encoded in the structure $\langle\Re,+, \cdot$, Integer $\rangle$, we conclude:

Lemma 8: The structure $\left\langle\mathcal{U} \cup \mathcal{P} \cup \Re \cup \Re^{\omega}\right.$, C,Convex,InPt,BdPt,Coors,,$+ \cdot$, Integer, []) $\rangle$ is definable in $\langle\mathcal{U}, \mathrm{C}$, Convex $\rangle$ under an encoding which is the identity over all of $\mathcal{U}$.

Next we show that infinite sequences of points, the indexing function on such sequences, and the closure function mapping an infinite sequence of points to a region, can all be represented. We 
will encode an infinite sequence of points as infinite sequences of pairs of reals, together with a coordinate system, where the sequences are the $\mathrm{x}$ - and $\mathrm{y}$-coordinates respectively of the points.

PtSeqIndex $(\hat{c}, \mathbf{o}, \mathbf{i}, \mathbf{j} ; ; \bar{k}, \mathbf{p})$ : The point $\mathbf{p}$ is the $k$ th element of the point sequence represented by the infinite sequence $\hat{c}$ of pairs of reals.

(3.37) PtSeqIndex $(\hat{c}, \bar{k}, \mathbf{p} ; \mathbf{o}, \mathbf{i}, \mathbf{j}) \equiv \exists_{\bar{x}, \bar{y}} \operatorname{PairIndex}(\hat{c}, \bar{k}, \bar{x}, \bar{y}) \wedge \operatorname{Coors}(\mathbf{p}, \bar{x}, \bar{y} ; \mathbf{o}, \mathbf{i}, \mathbf{j})$.

PtInClosure $(\mathbf{p}, \hat{c} ; \mathbf{o}, \mathbf{i}, \mathbf{j})$ : Point $p$ is in the closure of point sequence $\langle\hat{c}, \mathbf{o}, \mathbf{i}, \mathbf{j}\rangle$.

(3.38) PtInClosure $(\mathbf{p}, \hat{c} ; \mathbf{o}, \mathbf{i}, \mathbf{j}) \equiv$

$\exists_{\bar{u}, \bar{v}} \operatorname{Coors}(\mathbf{p}, \bar{u}, \bar{v} ; \mathbf{o}, \mathbf{i}, \mathbf{j}) \wedge$

$\forall_{\bar{e}>0} \exists_{\bar{k}, \bar{x}, \bar{y}}$ PairIndex $(\hat{c}, \bar{k}, \bar{x}, \bar{y}) \wedge|(\bar{u}-\bar{x})|+|(\bar{v}-\bar{y})|<\bar{e}$.

RegClosure $(\hat{c}, r ; \mathbf{o}, \mathbf{i}, \mathbf{j})$ : The closure of the sequence of points $\langle c, \mathbf{o}, \mathbf{i}, \mathbf{j}\rangle$ is region $r \in \mathcal{U}$.

(3.39) RegClosure $(\hat{c}, r ; \mathbf{o}, \mathbf{i}, \mathbf{j}) \equiv \forall_{\mathbf{p}} \operatorname{InPt}(\mathbf{p}, r) \Leftrightarrow \operatorname{PtInClosure}(\mathbf{p}, \hat{c} ; \mathbf{o}, \mathbf{i}, \mathbf{j})$.

Lemma 9: The structure $\left\langle\mathcal{U} \cup \mathcal{P} \cup \mathcal{P}^{\omega} \cup \Re \cup \Re^{\omega}\right.$, C,Convex,InPt,BdPt,Coors,,$+ \cdot$, Integer, [], PtInClosure, RegClosure $\rangle$ is definable in $\langle\mathcal{U}, \mathrm{C}$, Convex $\rangle$ under a mapping which is the identity over all of $\mathcal{U}$.

We now define an analytical relation over the set of closed regions: ${ }^{2}$

Definition 4: Let $\mathcal{C}$ be a coordinate system (i.e. a one-to-one mapping from points in the plane onto pairs of real numbers). Let $\mathcal{D}$ be the set of closed regions in the plane. For any pair of real numbers $\langle\bar{x}, \bar{y}\rangle$, let $\operatorname{At}(\bar{x}, \bar{y}, \mathcal{C})$ be the point in the plane with coordinates $\langle\bar{x}, \bar{y}\rangle$ in $\mathcal{C}$. For any sequence of real numbers $\hat{q} \in \Re^{\omega}$ let Closure $(\hat{q}, \mathcal{C})$ be the closure of the set of points $\{\operatorname{At}(\hat{q}[1], \hat{q}[2], \mathcal{C}), \operatorname{At}(\hat{q}[3], \hat{q}[4], \mathcal{C})$ $\ldots\}$. An $m$-place relation $\Phi\left(r_{1} \ldots r_{m}\right)$ over $\mathcal{D}$ is analytical with respect to $\mathcal{C}$ if the relation

$\Theta\left(\hat{q}_{1} \ldots \hat{q}_{m}\right) \equiv \Phi\left(\operatorname{Closure}\left(\hat{q}_{1}, \mathcal{C}\right) \ldots \operatorname{Closure}\left(\hat{q}_{m}, \mathcal{C}\right)\right)$

is an analytic relation over the $m$ sequences of real numbers $\hat{q}_{1} \ldots \hat{q}_{m}$; that is, the relation $\Theta$ is definable in the structure $\left\langle\Re, \Re^{\omega},+, \cdot\right.$, Integer, [] $\left.]\right\rangle$.

Theorem 1: Let $\mathcal{U}$ be a universe of closed regions in the plane that includes all simple polygons. Let $\Phi\left(r_{1} \ldots r_{m}\right)$ be a relation over regions in $\mathcal{U}$ that is analytical with respect to some coordinate system $\mathcal{C}$ and that is invariant under affine transformation. Then $\Phi$ is definable in $\langle\mathcal{U}, \mathrm{C}$, Convex $\rangle$.

Proof: Suppose that $\Phi$ is analytical with respect to coordinate system $\mathcal{C}$ and invariant under affine transformations. Let points $\mathbf{O}, \mathbf{I}, \mathbf{J}$ be respectively the origin, the point $\langle 1,0\rangle$ and the point $\langle 0,1\rangle$ in the coordinate system $\mathcal{C}$. Thus, $\operatorname{RegClosure}(\hat{q}, r, \mathbf{O}, \mathbf{I}, \mathbf{J})$ holds over $\mathcal{U}$ if and only if $\operatorname{Closure}(\hat{q}, \mathcal{C})=r$ and $r \in \mathcal{U}$. By definition 4, the relation $\Theta\left(\hat{q}_{1} \ldots \hat{q}_{m}\right) \equiv \Phi\left(\operatorname{Closure}\left(\hat{q}_{1}, \mathcal{C}\right) \ldots \operatorname{Closure}\left(\hat{q}_{m}, \mathcal{C}\right)\right)$ is an analytical function of the sequences of real numbers $\hat{q}_{1} \ldots \hat{q}_{m}$. By definition 1 and lemma 4 , this means that there exists a formula $\alpha\left(\hat{q}_{1} \ldots \hat{q}_{m}\right)$ that defines $\Phi$ over the structure $\left\langle\Re \cup \Re^{\omega},+, \cdot\right.$, Integer, [] Since $\Phi$ is invariant under affine transformations, it follows that $\Theta$ defines the same relation over sequences of coordinates regardless of the choice of affine coordinate system. Hence, for $r_{1} \ldots r_{m} \in \mathcal{U}$, the relation $\Phi\left(r_{1} \ldots r_{m}\right)$ holds if and only if

$$
\exists_{\hat{q}_{1} \ldots \hat{q}_{m}, \mathbf{o}, \mathbf{i}, \mathbf{j}} \operatorname{RegClosure}\left(\hat{q}_{1}, r_{1}, \mathbf{o}, \mathbf{i}, \mathbf{j}\right) \wedge \ldots \operatorname{Reg} \operatorname{Closure}\left(\hat{q}_{m}, r_{m}, \mathbf{o}, \mathbf{i}, \mathbf{j}\right) \wedge \alpha\left(\hat{q}_{1} \ldots \hat{q}_{m}\right) .
$$

As this last formula is a first-order formula over the structure

$\left\langle\mathcal{U} \cup \mathcal{P} \cup \mathcal{P}^{\omega} \cup \Re \cup \Re^{\omega}\right.$, C,Convex,InPt,BdPt,Coors,,$+ \cdot$, Integer, [], PtInClosure, RegClosure $\rangle$, by lemma 9 there exists a first-order formula over the structure $\langle\mathcal{U}, \mathrm{C}, \mathrm{Convex}\rangle$ that defines the same relation over $r_{1} \ldots r_{m}$. I

In contrast it may be noted that, working forward from the construction in [19], it is possible to prove a lemma that is almost, but not quite, analogous to lemma 9 for the structure $\langle\mathcal{U}, \mathrm{C}\rangle$, if $\mathcal{U}$ is the set of all regular regions. The result there, described in section 1 of this paper, shows that integers,

\footnotetext{
${ }^{2}$ As far as I know, this terminology and definition is original here. It seems to me the natural extension of the notion of an analytical relation to the space of geometric regions, though one could certainly wish for a more elegant definition.
} 
sequences of integers, addition, and multiplication, can be encoded in $\langle\mathcal{U}, \mathrm{C}\rangle$; one can continue on, as we have done here, and encode reals as bitstrings, points as pairs of reals, sequences of points as sequences of reals, and, as in lemma 11 (section 7) one may further encode closed regions as the closure of sequences of points. However, the difference is that this encoding is not the identity over the set of regions $\mathcal{U}$ and that, since apparently there is no way to define a coordinate system, there is no way to reconnect the closure of the sequence of points back to the starting region. Thus, one can define the relation "RegClosure $(\hat{q}, r)$ " between a series of reals $\hat{q}$ and an encoding of a region $r$, but not between $\hat{q}$ and the original region. Hence the proof of the analogue of Theorem 1 breaks down at the very last step; there is an encoding of $\Theta$ in $\langle\mathcal{U}, \mathrm{C}\rangle$, but it does not define the relation $\Phi$; it defines a different relation. This is why the result of (Schaefer and Stefankovic, 2004) is a complexity result but not an expressivity result.

Theorem 1 can be turned into a biconditional, if $\mathcal{U}$ is (very reasonably) required to be itself an analytical class, closed under affine transformation.

Lemma 10: The relations $\mathrm{C}(x, y)$ and $\operatorname{Convex}(x)$, over the space of closed regions, are analytical (as defined in definition 4).

Proof: Let $\mathcal{C}$ be a coordinate system. For any $\bar{x}, \bar{y}$, the relation InClosure $(\hat{q}, \bar{x}, \bar{y})$ (coordinate pair $\langle\bar{x}, \bar{y}\rangle$ is in the closure of $\hat{q}$ ) can be defined over the structure $\left\langle\Re, \Re^{\omega},+, \cdot\right.$, Integer, [] $\rangle$ by the following formula:

$$
\text { (3.40) } \forall_{\bar{e}>0} \exists_{\bar{k}} \operatorname{Integer}(\bar{k}) \wedge|\hat{q}[2 k]-x|+|\hat{q}[2 k+1]-y|<\bar{e} \text {. }
$$

The relation over $\hat{q}, \hat{r}, \mathrm{C}(\operatorname{Closure}(\hat{q}, \mathcal{C}), \operatorname{Closure}(\hat{r}, \mathcal{C}))$ can then be defined as

$$
\exists_{\bar{x}, \bar{y}} \text { InClosure }(\hat{q}, \bar{x}, \bar{y}) \wedge \operatorname{InClosure}(\hat{r}, \bar{x}, \bar{y}) \text {. }
$$

The property of $\hat{q}$, Convex $(\operatorname{Closure}(\hat{q}, \mathcal{C}))$ can be defined by the formula,

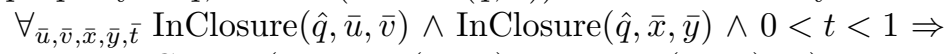
InClosure $(\hat{q}, t \cdot \bar{u}+(1-t) \cdot \bar{x}, t \cdot \bar{v}+(1-t) \cdot \bar{y})$.

Corollary 2: Let $\mathcal{U}$ and $\Phi$ be as in Theorem 1. Assume further that $\mathcal{U}$ is an analytic class of regions and is closed under affine transformations. Then $\Phi$ is first-order definable in $\langle\mathcal{U}, \mathrm{C}$, Convex $\rangle$ if and only if $\Phi$ is analytical and invariant under affine transformations.

Proof: The implication right to left is Theorem 1. Since $\mathrm{C}(x, y)$, Convex $(x)$, and $\mathcal{U}$ are analytical and invariable under affine transformation, it is trivial that any relation defined by a first-order formula over them must be analytical.

\section{Language of Distance}

In this section we deal with the structure over a domain $\mathcal{U}$ of regions with the single three-place relation Closer $(x, y, z)$, region $x$ is closer to region $y$ than it is to region $z$. As in section 3 we will allow the universe $\mathcal{U}$ to be any collection of closed regions that contains all simple solid polygons. Our approach will be to show that the structure $\langle\mathcal{U}, \mathrm{C}$, Convex $\rangle$ can be defined in $\langle\mathcal{U}$,Closer $\rangle$, but that in the latter structure one can additionally constrain a coordinate system to be orthogonal.

$\mathrm{C}(x, y)$ : Regions $x$ and $y$ are connected if nothing is closer to $y$ than $x$.

(4.1) $\mathrm{C}(x, y) \equiv \neg \exists_{z} \operatorname{Closer}(x, z, y)$.

Again, we can use definitions 3.1 and 3.2 to define $\mathrm{P}(x, y)$ and $\mathrm{PP}(x, y)$ in terms of $\mathrm{C}$.

AllCloser $(a, b, c)$ : All of region $b$ is closer to $a$ than $c$ is to $a$.

(4.2) $\operatorname{AllCloser}(a, b, c) \equiv \forall_{z} \mathrm{C}(z, b) \Rightarrow \operatorname{Closer}(a, z, c)$ 


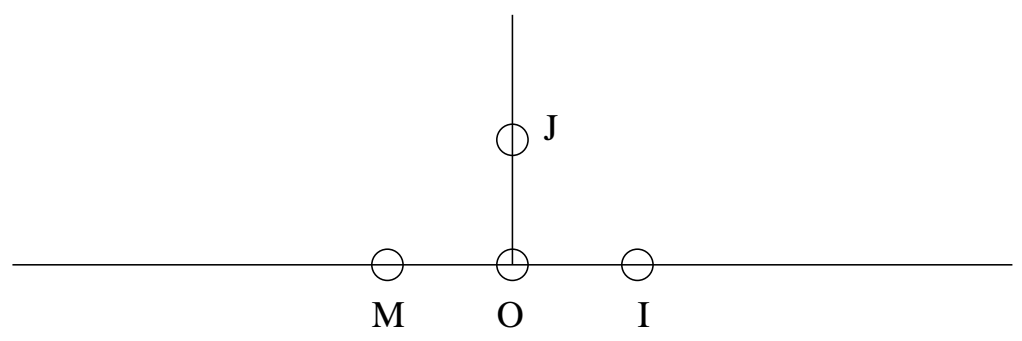

Figure 5: Orthogonal Coordinate System

InInterior $(a, b)$ - Region $a$ is in the interior of $b$ if every region $c$ that is entirely sufficiently close to $a$ is part of $b$.

(4.3) InInterior $(a, b) \equiv \exists_{d} \neg \mathrm{C}(d, a) \wedge \forall_{c} \operatorname{AllCloser}(a, c, d) \Rightarrow \mathrm{P}(c, b)$.

Regular $(b): b$ is regular if, for every region $c$ that is connected to $b$ there exist regions $a$ in the interior of $b$ that are arbitrarily close to $c$.

(4.4) Regular $(b) \equiv$

$$
\forall_{c, d}[\mathrm{C}(c, b) \wedge \neg \mathrm{C}(c, d)] \Rightarrow \exists_{a} \operatorname{Closer}(c, a, d) \wedge \operatorname{InInterior}(a, b) .
$$

Having defined regular regions, we can now define points as in definitions 3.9-3.14, replacing the predicate "ConvexSolid" throughout by "Regular". As in section 3 , we augment the universe $\mathcal{U}$ to include points, indicated with bold-face variables, and add the predicate "InPt".

$\operatorname{PtCloser}(\mathbf{p}, \mathbf{q}, \mathbf{r})$ : Point $\mathbf{p}$ is closer to $\mathbf{q}$ than to $\mathbf{r}$.

(4.5) $\operatorname{PtCloser}(\mathbf{p}, \mathbf{q}, \mathbf{r}) \equiv$

$$
\begin{aligned}
\forall_{a, b, c}[\operatorname{Regular}(a) & \wedge \operatorname{InPt}(\mathbf{p}, a) \wedge \operatorname{Regular}(b) \wedge \operatorname{InPt}(\mathbf{q}, b) \wedge \\
\operatorname{Regular}(c) & \wedge \operatorname{InPt}(\mathbf{r}, c)] \Rightarrow \\
\exists_{d, e, f} \mathrm{P}(d, a) & \wedge \mathrm{P}(e, b) \wedge \mathrm{P}(f, c) \wedge \operatorname{Closer}(d, e, f) .
\end{aligned}
$$

$\operatorname{Order}(\mathbf{p}, \mathbf{q}, \mathbf{r})$ : Points $\mathbf{p}, \mathbf{q}, \mathbf{r}$ lie in that order (non-strictly) on a line, if no point $\mathbf{z}$ is closer to both $\mathbf{p}$ and $\mathbf{r}$ than $\mathbf{q}$.

(4.5) $\operatorname{Order}(\mathbf{p}, \mathbf{q}, \mathbf{r}) \equiv \neg \exists_{\mathbf{z}} \operatorname{PtCloser}(\mathbf{p}, \mathbf{z}, \mathbf{q}) \wedge \operatorname{PtCloser}(\mathbf{r}, \mathbf{z}, \mathbf{q})$.

Convex $(a): a$ is convex.

(4.6) $\operatorname{Convex}(a) \equiv \forall_{\mathbf{p}, \mathbf{q}, \mathbf{r}}[\operatorname{InPt}(\mathbf{p}, a) \wedge \operatorname{InPt}(\mathbf{r}, a) \wedge \operatorname{Order}(\mathbf{p}, \mathbf{q}, \mathbf{r})] \Rightarrow \operatorname{InPt}(\mathbf{q}, a)$

Having defined $\mathrm{C}$ and Convex in $\langle\mathcal{U}$,Closer $\rangle$, we can now import all the results of section 3 . All that remains to be done is to show that one can define orthogonal coordinate systems, not just affine coordinate systems.

$\operatorname{EqPtDist}(\mathbf{p}, \mathbf{q}, \mathbf{r}): \mathbf{q}$ and $\mathbf{r}$ are equidistant from $\mathbf{p}$.

(4.7) $\operatorname{EqPtDist}(\mathbf{p}, \mathbf{q}, \mathbf{r}) \equiv \neg \operatorname{PtCloser}(\mathbf{p}, \mathbf{q}, \mathbf{r}) \wedge \neg \operatorname{PtCloser}(\mathbf{p}, \mathbf{r}, \mathbf{q})$.

OrthCoordSys $(\mathbf{o}, \mathbf{i}, \mathbf{j})$ : Points $\mathbf{o}, \mathbf{i}, \mathbf{j}$ defines an orthogonal coordinate system (Figure 5).

(4.8) OrthCoordSys $(\mathbf{o}, \mathbf{i}, \mathbf{j}) \equiv$ $\operatorname{AfCoordSys}(\mathbf{o}, \mathbf{i}, \mathbf{j}) \wedge \operatorname{EqPtDist}(\mathbf{o}, \mathbf{i}, \mathbf{j}) \wedge \exists_{m} \operatorname{EqualVec}(\mathbf{m}, \mathbf{o} ; \mathbf{o}, \mathbf{i}) \wedge \operatorname{EqPtDist}(\mathbf{j}, \mathbf{i}, \mathbf{m})$.

Theorem 3: Let $\mathcal{U}$ be a universe of closed regions in the plane that includes all simple polygons. Let $\Phi\left(r_{1} \ldots r_{m}\right)$ be a relation over regions in $\mathcal{U}$ that is analytical with respect to some coordinate system $\mathcal{C}$ and that is invariant under orthogonal transformations. Then $\Phi$ is definable in $\langle\mathcal{U}, \mathrm{C}, \mathrm{Convex}\rangle$.

Proof: Exactly analogous to the proof of Theorem 1. 
Corollary 4: Let $\mathcal{U}$ and $\Phi$ be as in Theorem 3. Assume further that $\mathcal{U}$ is an analytical class of regions and is closed under orthogonal transformations. Then $\Phi$ is first-order definable in $\langle\mathcal{U}$,Closer $\rangle$ if and only if $\Phi$ is analytical and invariant under orthogonal transformations.

Proof: Exactly analogous to the proof of Theorem 2.

\section{One Dimensional Geometry}

The constructions in previous sections work identically in Euclidean space of any dimension $k \geq 2$. The only changes that need to be made is that a coordinate system is specified by $k+1$ points; that a point has $k$ coordinates; and that "polygon" in the statement of the theorems must be replaced by " $k$-dimensional polyhedron", defined as a bounded, regular $k$-dimensional region whose boundary is the union of finitely many faces, each of which is a subset of a $k-1$ dimensional hyperplane.

The geometry of the one-dimensional line, however, is substantially different; many of the definitions can be made much simpler, but, more importantly, some key definitions no longer work. In particular, definitions 3.19 of "EqualVec", in terms of parallelograms, and 3.24 of "Ratio", in terms of similar parallelograms, cannot be made to work in one dimension. The predicate "Convex $(x)$ " is useless in one-dimension; a convex region is just a connected interval. Finally, in one-dimensional geometry, we cannot do without disconnected regions; if the universe of regions is restricted to connected regions (i.e. intervals), then most reasonable predicates, such as "Closer" can be computed just in terms of sums and differences over the coordinates of the endpoints; so the theory amounts to the theory of the real numbers with addition, which is an inexpressive and easily decidable theory.

Define a simple interval in the real line to be a closed bounded interval that is not a single point, and define a simple set to be the union of finitely many simple intervals. Let $\mathcal{U}$ be any universe of closed sets in the real line that includes all simple sets. Let Closer $(x, y, z)$ be the relation over regions, " $x$ is closer to $y$ than to $z$." We will show that any analytical relation over $\mathcal{U}$ that is invariant under linear transformation is first-order definable over $\langle\mathcal{U}$, Closer $\rangle$.

Begin by defining the relation $\mathrm{C}(x, y)$ as in definition 4.1 , and then defining the relations $\mathrm{P}(x, y)$, $\mathrm{PP}(x, y)$, Union $(x, y, z), \operatorname{Conn}(x)$ as in definitions 3.1, 3.2, 3.4, 3.5.

Interval $(x): x$ is a simple interval if it is a connected set with at least two points.

(5.1) Interval $(x) \equiv \operatorname{Conn}(x) \wedge \exists_{y, z} \mathrm{P}(y, x) \wedge \mathrm{P}(z, x) \wedge \neg \mathrm{C}(y, z)$.

$\mathrm{O}(x, y): x$ and $y$ overlap.

(5.2) $\mathrm{O}(x, y) \equiv \exists_{z} \operatorname{Interval}(z) \wedge \mathrm{P}(z, x) \wedge \mathrm{P}(z, y)$.

$\mathrm{EC}(x, y): x$ and $y$ are externally connected.

(5.3) $\mathrm{EC}(x, y) \equiv \mathrm{C}(x, y) \wedge \neg \mathrm{O}(x, y)$.

TPPInt $(x, y)$ : Interval $x$ is a tangential partial part of interval $y$.

(5.4) $\operatorname{TPPInt}(x, y) \equiv$ $\operatorname{Interval}(x) \wedge \operatorname{Interval}(y) \wedge \operatorname{PP}(x, y) \wedge \exists_{z} \operatorname{EC}(z, x) \wedge \operatorname{EC}(z, y)$.

Component $(x, y): x$ is a connected component of $y$ if it is a maximal connected subset of $y$.

(5.5) Component $(x, y) \equiv \mathrm{P}(x, y) \wedge \operatorname{Conn}(x) \wedge \neg \exists_{z} \mathrm{PP}(x, z) \wedge \mathrm{P}(z, y) \wedge \operatorname{Conn}(z)$.

SameDist $(a, b, c)$ : Region $a$ is equidistant from $b$ and $c$.

(5.6) SameDist $(a, b, c) \equiv \neg \operatorname{Closer}(a, b, c) \wedge \neg \operatorname{Closer}(a, c, b)$.

Concentric $1(i, j)$ : Interval $i$ is inside interval $j$ and has the same midpoint if $i$ is equidistant from every region EC to $j$. (Figure 6.) 


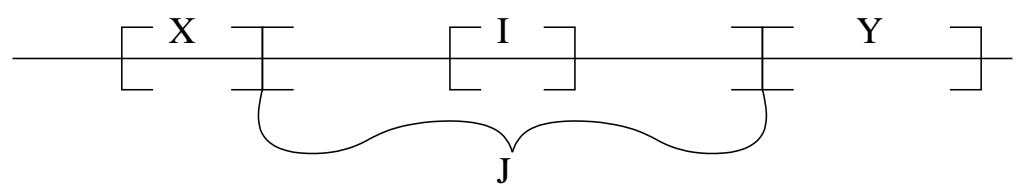

Figure 6: Concentric Intervals

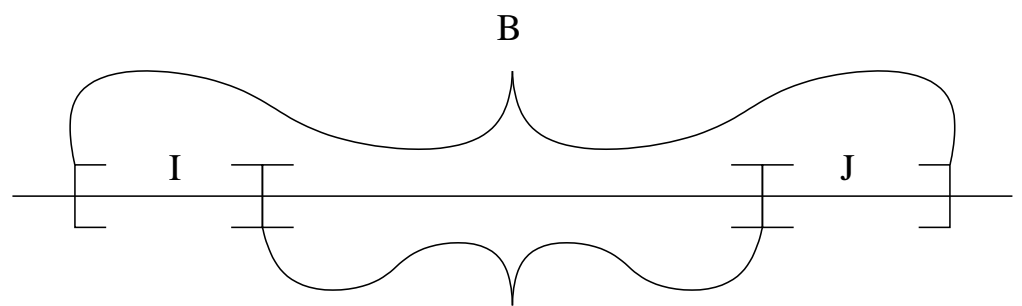

A

Figure 7: Two intervals have the same lengths

(5.7) Concentric $1(i, j) \equiv$

$\operatorname{Interval}(i) \wedge \operatorname{Interval}(j) \wedge \mathrm{P}(i, j) \wedge \forall_{x, y}[\mathrm{EC}(x, j) \wedge \mathrm{EC}(y, j)] \Rightarrow \operatorname{SameDist}(i, x, y)$.

SameLengthDC $(i, j)$ : Intervals $i$ and $j$ are disconnected and have the same length (Figure 7).

(5.8) SameLengthDC $(i, j) \equiv$ Interval $(i) \wedge \operatorname{Interval}(j) \wedge \neg \mathrm{C}(i, j) \wedge$

$\exists_{a, b} \operatorname{Concentric} 1(a, b) \wedge \operatorname{EC}(i, a) \wedge \operatorname{EC}(j, a) \wedge \operatorname{TPPInt}(i, b) \wedge \operatorname{TPPInt}(j, b)$.

SameLength $(i, j)$ : Intervals $i$ and $j$ have the same length.

(5.9) SameLength $(i, j) \equiv \exists_{k}$ SameLength1 $(i, k) \wedge \operatorname{SameLength1}(k, j)$.

A point is represented by any two intervals that are EC.

IsPoint $(a, b)$ : Intervals $a$ and $b$ define a point.

(5.10) $\operatorname{IsPoint}(a, b) \equiv \operatorname{ECInt}(a, b)$.

SamePoint $(a, b ; c, d): a, b$ determine the same point as $c, d$.

(5.11) SamePoint $(a, b ; c, d) \equiv$

$\operatorname{IsPoint}(a, b) \wedge \operatorname{IsPoint}(c, d) \wedge$

$[[\operatorname{ECInt}(a, c) \wedge \operatorname{ECInt}(b, d)] \vee[\operatorname{ECInt}(a, d) \wedge \operatorname{ECInt}(b, c)]]$

The relations $\operatorname{InPt}(a, b ; r)$ and $\operatorname{BdPt}(a, b ; r)$ can be defined using definitions 3.12 and 3.14.

As in previous sections, we now add points to our universe and our language. Distances between points are measured by the lengths of the intervals that lie between them.

OppEnds $(\mathbf{p}, \mathbf{q}, r)$ : Points $\mathbf{p}$ and $\mathbf{q}$ are at opposite ends of interval $r$.

(5.12) OppEnds $(\mathbf{p}, \mathbf{q}, r) \equiv \operatorname{BdPt}(\mathbf{p}, r) \wedge \operatorname{BdPt}(\mathbf{q}, r) \wedge \mathbf{p} \neq \mathbf{q}$.

$\operatorname{PtDistEq}(\mathbf{p}, \mathbf{q} ; \mathbf{r}, \mathbf{s}):|\mathbf{p q}|=|\mathbf{r s}|$.

(5.13) $\operatorname{PtDistEq}(\mathbf{p}, \mathbf{q} ; \mathbf{r}, \mathbf{s}) \equiv$

$[\mathbf{p}=\mathbf{q} \wedge \mathbf{r}=\mathbf{s}] \vee$

$\exists_{a, b} \operatorname{SameLength}(a, b) \wedge \operatorname{OppEnds}(\mathbf{p}, \mathbf{q}, a) \wedge \operatorname{OppEnds}(\mathbf{r}, \mathbf{s}, b)$.

$\operatorname{VecEq}(\mathbf{p}, \mathbf{q} ; \mathbf{r}, \mathbf{s}): \overrightarrow{p q}=\overrightarrow{r s}$.

(5.14) $\operatorname{VecEq}(\mathbf{p}, \mathbf{q} ; \mathbf{r}, \mathbf{s}) \equiv \operatorname{PtDistEq}(\mathbf{p}, \mathbf{q} ; \mathbf{r}, \mathbf{s}) \wedge \operatorname{PtDistEq}(\mathbf{p}, \mathbf{r} ; \mathbf{q}, \mathbf{s})$. 
We represent an integer $k \geq 1$ by a simple region with $k$ connected components. The relation " $a$ and $b$ have the same number of connected components" is defined as a combination of two relations. First, if each connected component of $b$ is a subset of exactly one component of $a$, and each component of $a$ contains exactly one component of $b$, then $a$ and $b$ have the same number of components. In this case we say that $b$ is a shrinking of $a$. Second, if each component of $p$ is the same length as one component of $q$, and vice versa, then $a$ and $b$ have the same number of components; in this case we say that $q$ is a rearrangement of $p$. Finally, for any simple regions $a$ and $b, a$ and $b$ have the same number of components if and only if there exist $p$ and $q$ such that $p$ is a shrinking of $a, q$ is a shrinking of $b$, and $q$ is a rearrangement of $p$. (The construction here is similar to that of Grzegorczyk [10].)

$\operatorname{Bounded}(r): r$ is a bounded region.

(5.15) Bounded $(r) \equiv \exists_{x} \forall_{a, b} \mathrm{P}(a, r) \wedge \mathrm{P}(b, r) \Rightarrow \operatorname{Closer}(a, b, x)$.

Whole $(r): r$ is a simple region, and thus represents a whole number.

(5.16) Whole $(r) \equiv$

$$
\operatorname{Bounded}(r) \wedge \forall_{\mathbf{p}} \operatorname{InPt}(\mathbf{p}, r) \Rightarrow \exists_{z} \operatorname{PP}(z, r) \wedge \operatorname{Interval}(z) \wedge \operatorname{InPt}(\mathbf{p}, z) .
$$

Shrink $(a, p)$ : $a$ can be shrunk to $p$, preserving the number of connected components.

(5.17) $\operatorname{Shrink}(a, p) \equiv$ $\operatorname{Whole}(a) \wedge \operatorname{Whole}(p) \wedge$

$\left[\forall_{x} \operatorname{Component}(x, a) \Rightarrow \exists_{y}^{1} \operatorname{Component}(y, p) \wedge \mathrm{P}(y, x)\right] \wedge$

$\left[\forall y\right.$ Component $\left.(y, p) \Rightarrow \exists_{x}^{1} \operatorname{Component}(x, a) \wedge \mathrm{P}(y, x)\right]$

Rearrange $(p, q): q$ is a rearrangement of $q$.

(5.18) Rearrange $(p, q) \equiv$

$$
\begin{aligned}
& {\left[\forall_{x} \text { Component }(x, p) \Rightarrow \exists_{z}^{1} \operatorname{Component}(z, q) \wedge \operatorname{SameLength}(x, z)\right] \wedge} \\
& {\left[\forall_{z} \text { Component }(z, q) \Rightarrow \exists_{x}^{1} \operatorname{Component}(x, p) \wedge \operatorname{SameLength}(x, z)\right]}
\end{aligned}
$$

SameNum $(a, b)$ : Regions $a$ and $b$ have the same number of connected components.

(5.19) $\operatorname{SameNum}(a, b) \equiv$ $\exists_{p, q} \operatorname{Shrink}(a, p) \wedge \operatorname{Shrink}(b, q) \wedge$ Rearrange $(p, q)$.

Plus $(x, y, z)$ and $\operatorname{Times}(x, y, z)$ can now be defined over the whole numbers:

The sum of two numbers is just their disjoint union.

(5.20) $\operatorname{Plus}(a, b, c) \equiv$

$\exists_{d, e} \operatorname{SameNum}(b, d) \wedge \neg \mathrm{C}(a, d) \wedge \operatorname{Union}(a, d, e) \wedge \operatorname{SameNum}(e, c)$.

The number $r$ is the product $p \cdot q$ if $r \subset p$ and every connected component of $p$ contains exactly $q$ components of $r$.

$\operatorname{Times}(p, q, r): p \cdot q=r$.

(5.21) $\operatorname{Times}(p, q, r) \equiv$ $\exists_{w} \operatorname{SameNum}(w, r) \wedge \mathrm{P}(w, p) \wedge$

$\forall_{c, d}$ Component $(c, p) \wedge \operatorname{Intersect}(r, c, d) \Rightarrow \operatorname{SameNum}(d, q)$.

We represent real numbers using a point $\mathbf{p}$ on a coordinate axis $\mathbf{o}, \mathbf{u}$, as in section 3 . A real number $\mathbf{p}$ is positive if there is an interval that contains $\mathbf{p}$ and $\mathbf{u}$ but not $\mathbf{o}$.

$\operatorname{CoordSys}(\mathbf{o}, \mathbf{u}): \mathbf{o}, \mathbf{u}$ determine a coordinate system.

(5.22) CoordSys $(\mathbf{o}, \mathbf{u}) \equiv \mathbf{o} \neq \mathbf{u}$. 


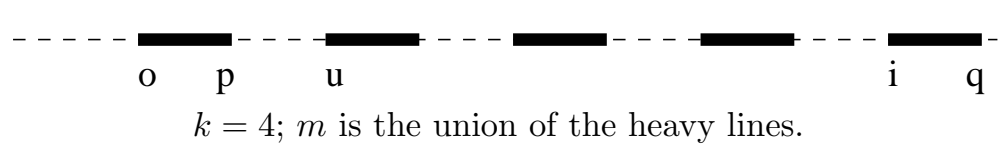

Figure 8: Integer to Real Conversion

$\operatorname{RPlus}(\mathbf{p}, \mathbf{q}, \mathbf{r} ; \mathbf{o}, \mathbf{u}): \mathbf{p}+\mathbf{q}=\mathbf{r}$, as reals in coordinate system $\mathbf{o}, \mathbf{u}$.

(5.23) RPlus $(\mathbf{p}, \mathbf{q}, \mathbf{r} ; \mathbf{o}, \mathbf{u}) \equiv \operatorname{VecEq}(\mathbf{o}, \mathbf{p} ; \mathbf{q}, \mathbf{r})$.

$\operatorname{Pos}(\mathbf{p} ; \mathbf{o}, \mathbf{u}): p$ is a positive real relative to $\mathbf{o}, \mathbf{u}$.

(5.24) $\operatorname{Pos}(\mathbf{p} ; \mathbf{o}, \mathbf{u}) \equiv$

$\exists_{z} \operatorname{Interval}(z) \wedge \operatorname{PtIn}(\mathbf{p}, z) \wedge \operatorname{PtIn}(\mathbf{u}, z) \wedge \neg \operatorname{PtIn}(\mathbf{o}, z)$

$\operatorname{Less}(\mathbf{p}, \mathbf{q} ; \mathbf{o}, \mathbf{u}): \mathbf{p}<\mathbf{q}$ relative to $\mathbf{o}, \mathbf{u}$.

(5.25) $\operatorname{Less}(\mathbf{p}, \mathbf{q} ; \mathbf{o}, \mathbf{u}) \equiv \exists_{z} \operatorname{Pos}(\mathbf{z} ; \mathbf{o}, \mathbf{u}) \wedge \operatorname{RPlus}(\mathbf{p}, \mathbf{z}, \mathbf{q} ; \mathbf{o}, \mathbf{u})$.

(5.26) $\operatorname{Leq}(\mathbf{p}, \mathbf{q} ; \mathbf{o}, \mathbf{u}) \equiv \operatorname{Less}(\mathbf{p}, \mathbf{q} ; \mathbf{o}, \mathbf{u}) \vee \mathbf{p}=\mathbf{q}$

To convert an integer $k \geq 1$, represented as a region with $k$ components, to the real number $\mathbf{k} ; \mathbf{o}, \mathbf{u}$, we construct a region $p$ whose $k+1$ components all have length $1 / 2$ and are separated by gaps of length $1 / 2$.

$\operatorname{Gap}(g, p): g$ is a gap in region $p$.

(5.27) $\operatorname{Gap}(g, p) \equiv$

$\neg \mathrm{O}(g, p) \wedge \operatorname{Interval}(g) \wedge$

$\exists_{i, j} \operatorname{Component}(i, p) \wedge \operatorname{Component}(j, p) \wedge i \neq j \wedge \operatorname{EC}(g, i) \wedge \operatorname{EC}(g, j)$.

$\operatorname{Ruler}(p): p$ is a region with equally spaced components and gaps.

(5.28) $\operatorname{Ruler}(p) \equiv$

$\forall_{x, y, g} \operatorname{Component}(x, p) \wedge \operatorname{Component}(y, p) \wedge \operatorname{Gap}(g, p) \Rightarrow$

$\operatorname{SameLength}(x, y) \wedge \operatorname{SameLength}(x, g)$.

$\operatorname{Succ}(p, q): q=p+1$.

(5.29) $\operatorname{Succ}(p, q) \equiv \exists_{r} \operatorname{Interval}(r) \wedge \operatorname{Plus}(p, r, q)$

$\operatorname{BdComponent}(\mathbf{p}, r): \mathbf{p}$ is an endpoint of one component of $r$.

(5.30) $\operatorname{BdComponent}(\mathbf{p}, r) \equiv \exists_{c} \operatorname{Components}(c, r) \wedge \operatorname{BdPt}(\mathbf{p}, c)$.

$\operatorname{Int2Real}(k, \mathbf{i} ; \mathbf{o}, \mathbf{u})$ : The real number $\mathbf{i} ; \mathbf{o}, \mathbf{u}$ corresponds to integer $k$ (Figure 8).

(5.31) $\operatorname{Int} 2 \operatorname{Real}(k, \mathbf{i} ; \mathbf{o}, \mathbf{u}) \equiv$

$\exists_{m, p, q} \operatorname{Succ}(k, m) \wedge \operatorname{Ruler}(m) \wedge$

$\operatorname{BdComponent}(\mathbf{o}, m) \wedge \operatorname{BdComponent}(\mathbf{u}, m) \wedge \operatorname{BdComponent}(\mathbf{i}, m) \wedge$

$\operatorname{BdComponent}(\mathbf{p}, m) \wedge \operatorname{BdComponent}(\mathbf{q}, m) \wedge$

$\operatorname{Less}(\mathbf{o}, \mathbf{p} ; \mathbf{o}, \mathbf{u}) \wedge \operatorname{Less}(\mathbf{p}, \mathbf{u} ; \mathbf{o}, \mathbf{u}) \wedge \operatorname{Less}(\mathbf{u}, \mathbf{i} ; \mathbf{o}, \mathbf{u}) \wedge \operatorname{Less}(\mathbf{i}, \mathbf{q} ; \mathbf{o}, \mathbf{u}) \wedge$

$\forall_{\mathbf{x}} \operatorname{BdComponent}(\mathbf{x}, m) \Rightarrow \mathbf{x}=\mathbf{o} \vee \mathbf{x}=\mathbf{p} \vee \mathbf{x}=\mathbf{q} \vee$

$[\operatorname{Leq}(\mathbf{u}, \mathbf{x} ; \mathbf{o}, \mathbf{u}) \wedge \operatorname{Leq}(\mathbf{x}, \mathbf{i} ; \mathbf{o}, \mathbf{u})]$

We define real ratio in terms of integer multiplication: For positive real $a, b, c, d$ on a line with origin $o, a b<c d$ if there is some scale $v$ in which $\lceil a\rceil\lceil b\rceil<\lfloor c\rfloor\lfloor d\rfloor$.

PosLessTimes $(\mathbf{a}, \mathbf{b}, \mathbf{c}, \mathbf{d} ; \mathbf{o}, \mathbf{u}): a b<c d$ where $a, b, c, d$ are positive real.

(5.32) PosLessTimes $(\mathbf{a}, \mathbf{b}, \mathbf{c}, \mathbf{d} ; \mathbf{o}, \mathbf{u}) \equiv$

$\exists_{e, f, g, h, i, j, k, \mathbf{v}, \mathbf{w}, \mathbf{x}, \mathbf{y}, \mathbf{z}} \operatorname{Times}(e, f, g) \wedge \operatorname{Times}(h, i, j) \wedge \operatorname{Plus}(g, k, j) \wedge \operatorname{Pos}(\mathbf{v} ; \mathbf{o}, \mathbf{u}) \wedge$

$\operatorname{Int} 2 \operatorname{Real}(e, \mathbf{w} ; \mathbf{o}, \mathbf{v}) \wedge \operatorname{Int} 2 \operatorname{Real}(f, \mathbf{x} ; \mathbf{o}, \mathbf{v}) \wedge$ 


$$
\begin{aligned}
& \operatorname{Int} 2 \operatorname{Real}(h, \mathbf{y} ; \mathbf{o}, \mathbf{v}) \wedge \operatorname{Int} 2 \operatorname{Real}(i, \mathbf{z} ; \mathbf{o}, \mathbf{v}) \wedge \\
& \operatorname{Less}(\mathbf{a}, \mathbf{w} ; \mathbf{o}, \mathbf{v}) \wedge \operatorname{Less}(\mathbf{b}, \mathbf{x} ; \mathbf{o}, \mathbf{v}) \wedge \operatorname{Less}(\mathbf{c}, \mathbf{y} ; \mathbf{o}, \mathbf{v}) \wedge \operatorname{Less}(\mathbf{d}, \mathbf{z} ; \mathbf{o}, \mathbf{v}) .
\end{aligned}
$$

Note: In the above definition $e \cdot f=g, h \cdot i=j$, and $g+k=j$; since $k>0$, we have $e \cdot f<g \cdot h$. In coordinate system $\mathbf{o , v}$ the point $\mathbf{w}$ corresponds to $e, \mathbf{x}$ to $f, \mathbf{y}$ to $h$ and $\mathbf{z}$ to $i$; thus $\mathbf{w} \cdot \mathbf{x}<\mathbf{y} \cdot \mathbf{z}$. Finally, $\mathbf{a}<\mathbf{w}, \mathbf{b}<\mathbf{x}, \mathbf{y}<\mathbf{c}$, and $\mathbf{z}<\mathbf{d}$, so $\mathbf{a} \cdot \mathbf{b}<\mathbf{c} \cdot \mathbf{d}$.

$\operatorname{PosTimes}(\mathbf{p}, \mathbf{q}, \mathbf{r} ; \mathbf{o}, \mathbf{u}): \mathbf{p} \cdot \mathbf{q}=\mathbf{r}$ relative to $\mathbf{o}, \mathbf{u}$ where $\mathbf{p}, \mathbf{q}, \mathbf{r}$ are positive.

(5.33) $\operatorname{PosTimes}(\mathbf{p}, \mathbf{q}, \mathbf{r} ; \mathbf{o}, \mathbf{u}) \equiv$

$\operatorname{Pos}(\mathbf{p} ; \mathbf{o}, \mathbf{u}) \wedge \operatorname{Pos}(\mathbf{q} ; \mathbf{o}, \mathbf{u}) \wedge$

$\neg \operatorname{PosLessTimes}(\mathbf{p}, \mathbf{q}, \mathbf{u}, \mathbf{r} ; \mathbf{o}, \mathbf{u}) \wedge \neg \operatorname{PosLessTimes}(\mathbf{u}, \mathbf{r}, \mathbf{p}, \mathbf{q} ; \mathbf{o}, \mathbf{u})$.

The extension of addition and multiplication to zero and negative reals is straightforward.

Theorem 5: Let $\mathcal{U}$ be a universe of closed sets in the line that includes all simple sets. Let $\Phi\left(r_{1} \ldots r_{m}\right)$ be a relation over regions in $\mathcal{U}$ that is analytical with respect to some coordinate system $\mathcal{C}$ and that is invariant under linear transformations. Then $\Phi$ is definable in $\langle\mathcal{U}$, Closer $\rangle$.

Proof: Exactly analogous to the proof of Theorem 1.

Corollary 6: Let $\mathcal{U}$ and $\Phi$ be as in Theorem 5. Assume further that $\mathcal{U}$ is an analytic class of regions and is closed under linear transformations. Then $\Phi$ is first-order definable in $\langle\mathcal{U}$,Closer $\rangle$ if and only if $\Phi$ is analytical and invariant under linear transformations.

Proof: Exactly analogous to the proof of corollary 2.

\section{Rational Polygons}

We now consider the corresponding structures where the domain is the class of polygons with vertices with rational coordinates, as advocated by Pratt [16].

Definition 5: A relation over the natural numbers is arithmetical if it is first-order definable in the structure $\langle\omega,+, \cdot\rangle$.

Definition 6: Assume a fixed coordinate system $\mathcal{C}$. Let $\mathcal{U}$ be the class of rational polygons; i.e. simple polygons whose vertices have rational coefficients in $\mathcal{C}$. Every rational point corresponds to a pair of rational and thus a quadruple of integers. Any $m$-sided rational polygon $r \in \mathcal{U}$ can be encoded as a single natural number by taking the sequence of vertices, encoding each vertex as a quadruple of integers, and then encoding the $4 m$-tuple of integers as a natural number using a standard computable encoding of finite sequences of integers ([12] p. 32). An relation $m$-place relation $\Phi\left(r_{1} \ldots r_{m}\right)$ over $\mathcal{U}$ is arithmetical if the corresponding relation over the encodings of $r_{1} \ldots r_{m}$ is arithmetical.

Theorem 5: In two and higher dimensional Euclidean space, if the universe of regions is restricted to the class of polygons with rational coefficients, then results exactly analogous to corollaries 2,4 , and 6 substituting "arithmetical" for "analytical", and restricting the affine/orthogonal transformations to those with rational coefficients. In one-dimensional space, if the universe of regions is restricted to finite unions of intervals with rational endpoints, then the result analogous to Theorem 4 holds, again substituting "arithmetical" for "analytical" and making the same restriction.

Proof sketch: To show that all relations that are first-order definable in these structures are arithmetical, note that all these relations between regions can be computed from the coefficients of their vertices; that these coefficients are rational numbers and thus pairs of integers; and that a finite sequence of integers can be encoded as a single integer.

To show that all arithmetical relations can be defined in these structures, note that all the con- 
structions of sections 3-5 can be carried out using rational polygons, except that those constructions which previously generated real numbers now generate rational numbers. Therefore, integers and integer addition and multiplication can be represented in all these structures; an affine coordinate system can be constructed in the structure with two-dimensional regions with relations $\mathrm{C}(x, y)$ and Convex $(x)$; and an orthogonal coordinate system can be generated in the structure with relation $\operatorname{Closer}(x, y, z)$.

\section{Some analytical relations over regions}

In this section we demonstrate that a number of more advanced concepts from geometry and analysis are analytical, in the sense of definition 4 . Since the proofs here are straightforward, and since they do not lead to any overarching general theorem, it will suffice just to sketch the constructions; the reader can easily fill in the details. First, we need a couple of general lemmas. Throughout this section let $\mathcal{S}$ be either the structure $\langle\mathcal{U}, \mathrm{C}$, Convex $\rangle$ or $\langle\mathcal{U}$, Closer $\rangle$.

Lemma 12: Let $\mathcal{D}$ be the class of closed regions in the plane. Then $\mathcal{D}$, and the relation $\operatorname{InPt}(\mathbf{p}, d)$, point $\mathbf{p}$ is in $d$, can be encoded in $\mathcal{S}$.

Proof: Encode a closed region $d \in D$ as a sequence of points $\hat{p}$ whose closure is equal to $d$. The relation InPt is then just the same as PtInClosure.

Lemma 13: The class $\mathcal{D}^{\omega}$ of infinite sequences of closed regions and the indexing function $\hat{d}[i]$ can be encoded in $\mathcal{S}$.

Proof: For each region $\hat{d}[i]$, pick a sequence of points that encodes $\hat{d}[i]$ and then dovetail the sequences.

Theorem 7: The relation "Homeomorphic $(p, q)$ " (regions $p$ and $q$ are homeomorphic) is first-order definable in the structure $\mathcal{S}$.

Proof: Since the relation is obviously invariant under affine transformations, by Theorems 1 and 3, it suffices to show that the relation is analytical. Any continuous function over region $p$ is determined by its value on a countable dense set of points in $p$. Hence, such a function $f$ can be encoded in terms of a sequence of points, $\mathbf{x}_{1}, f\left(\mathbf{x}_{1}\right), \mathbf{x}_{2}, f\left(\mathbf{x}_{2}\right), \ldots$, where $\mathbf{x}_{1}, \mathbf{x}_{2} \ldots$ is dense in $p$. Given such an encoding of $f$, it is easy to define the relations " $q$ is the image of $p$ under $f$ ", and " $f$ is a homeomorphism" in terms of the relations we have defined above. The relation " $p$ and $q$ are homeomorphic" is then defined as "there exists a homeomorphism $f$ such that $q=f(p)$."

Theorem 8: The relation "SemiAlgebraic $(r ; \mathbf{o}, \mathbf{i}, \mathbf{j})$ " (region $r$ is semi-algebraic relative to the coordinate system $\mathbf{o}, \mathbf{i}, \mathbf{j}$ ) is first-order definable in the structure $\mathcal{S}$.

Proof: By Theorems 1 and 3 it suffices to show that this relation is analytical. A closed region $p$ is semi-algebraic relative to coordinate system $\mathcal{C}$ if and only if there exists a sequence of multinomials $f_{1,1}(x, y) \ldots f_{1, k}(x, y) \ldots f_{m, 1}(x, y) \ldots f_{m, k}(x, y)$ with integer coefficients and exponents such that a point $\mathbf{p}$ is in $r$ if and only if the coordinates of $\mathbf{p}$ in $\mathcal{C}$ satisfy the constraint

$P(x, y) \equiv\left[f_{1,1}(x, y) \geq 0 \wedge \ldots \wedge f_{1, k}(x, y) \geq 0\right] \vee \ldots \vee\left[f_{m, 1}(x, y) \geq 0 \wedge \ldots \wedge f_{m, k}(x, y) \geq 0\right]$.

Each multinomial can be encoded as a sequence of integers, and therefore the entire constraint can be encoded as a sequence of integers. Given such an encoding, it is straightforward to define the relations "Sequence $\hat{s}$ is an encoding of an algebraic constraint" and "The constraint encoded by $\hat{s}$ holds on $x, y$ ". The property "Region $r$ is semi-algebraic relative to the coordinate system $\mathbf{o}, \mathbf{i}, \mathbf{j}$ " can then be defined as "There exists a sequence $\hat{s}$ which is an encoding of an algebraic constraint" etc.

Lemma 14: Let $r$ and $s$ be two bounded regions in the plane. The area of $r$ is greater than the 
area of $s$ if and only if there exist sequences of regions $r_{1} \ldots r_{m}$ and $s_{1} \ldots s_{m}$ such that:

- $r_{i}$ and $r_{j}$ do not overlap for $i \neq j$.

- $r_{i} \subset r$ for $i=1 \ldots m$.

- $s$ is a proper subset of $s_{1} \cup \ldots \cup s_{m}$.

- $s_{i}$ is congruent to $r_{i}$ for $i=1 \ldots m$.

Proof: If the conditions are satisfied then Area $(s)<\sum_{i} \operatorname{Area}\left(s_{i}\right)=\sum_{i} \operatorname{Area}\left(r_{i}\right) \leq \operatorname{Area}(r)$.

Conversely, suppose Area $(r)>\operatorname{Area}(s)$. Let $\epsilon=(\operatorname{Area}(r)-\operatorname{Area}(s)) / 2$. Then there exists $\delta>0$ such that if $G$ is any square grid of side $\delta$, the total area of the squares in $G$ that are completely inside $r$ is at least Area $(r)-\epsilon$, and the total areas of the squares in $G$ that overlap $s$ is at most Area $(s)+\epsilon$. Since all the squares have equal area, the number squares completely inside $r$ must be greater than the number of squares overlapping $s$. Hence, we may choose $r_{1} \ldots r_{m}$ to be all the squares in $G$ completely inside $r$ and $s_{1} \ldots s_{m}$ to be all the squares in $G$ that overlap $s$, plus an arbitrary choice of other squares to make up the count of $m$.

Theorem 9: The relation SameArea $(r, s)$ (regions $r$ and $s$ have the same area) is first-order definable in the structure $\mathcal{S}$.

Proof: Since the relation is invariant under affine transformation, by Theorems 1 and 3 it suffices to show that this relation is analytical. Using the results of section 3 and lemma 12, it is immediate that the conditions in lemma 13 are definable in $\mathcal{S}$, so the relation "The area of $r$ is greater than the area of $s "$ is definable.

If $r$ and $s$ may be unbounded, then they have the same area if and only if for all bounded $p \subset r$ there exists bounded $q \subset s$ such that $\operatorname{Area}(p)=\operatorname{Area}(q)$ and vice versa.

The reader can easily continue this list to show that many, many favorite geometric relations are analytical and therefore definable in these structures. Even relations that are often thought of as weird or pathological - "The projection of region $r$ is the Cantor set", "The boundary of region $r$ is nowhere differentiable," "The three-dimensional curve $c=\{\langle x, y, t\rangle\}$ where $\langle x(t), y(t)\rangle$ is a space-filling curve in 2D" etc. - are easily shown to be analytical, and therefore expressible if the universe $\mathcal{U}$ is defined to include these monsters.

\section{Related Work}

The study of geometric theories based around on extended regions began with de Laguna [7] and Whitehead [21] and was greatly advanced by Clarke [5].

Tarski [20] studied a language over a universe of regions with the two primitives $\mathrm{C}(x, y)$ and $\mathrm{S}(x)$ ( $x$ is a sphere); he was not precise as to the logic he has in mind, but his exposition includes second-order constructions (e.g. the definition of a point in terms of a set of concentric spheres.) This theory was made more formal by Bennett [1], who developed first- and second-order axiomatizations of this theory and proves that the axioms are categorical.

Grzegorczyk [10] proved that the first-order language over regions with the predicate $\mathrm{C}(x, y)$ is undecidable. A similar result was rediscovered independently, with a very different proof, by Dornheim [8]. Grzegorczyk encodes an integer $k$ as a region with $k$ connected components. The theorem holds even if the universe of regions is restricted to polygons (indeed, even if it is restricted to polygons with horizontal and vertical edges.) 
The undecidability result also holds if the universe of regions is restricted to convex polygons. The integer $k$ is encoded as a pair of convex polygons $A, B$ such that $A-B$ has $k$ connected components. Equality, addition, and multiplication of integers can then be defined as in definitions 5.19 through 5.21, replacing $\operatorname{SameLength}(x, z)$ in 5.18 with AffineTransform $(x, z)$ ( $x$ is an affine transformation of $z$.)

Randell, Cui, and Cohn [18] introduced the region connection calculus (RCC), and demonstrated that the RCC primitives and other important topological properties can be defined in the first-order logic over regular regions with the predicate $\mathrm{C}(x, y)$. The expressivity of this language was further studied by Gotts [9]. Pratt and Schoop [17] studied the expressivity of the language over the space of polygonal region with the primitive $\mathrm{C}(x, y)$ and the language over the same universe with the primitives Conn $(x)$ and $\mathrm{P}(x, y)$. They prove that both languages are strong enough to specify completely the topological relations among any $n$-tuple of regions. (More precisely, the first language can achieve this over the open plane; the second can achieve it only for regions within the closed plane.) The constraint language of RCC is known to be tractable [15].

Randell, Cui, and Cohn [18] also studied the first order language with predicate $\mathrm{C}(x, y)$ and Convex $(x)$, and presented some useful qualitative properties that could be defined in that language. Pratt [16] proved that this language is powerful enough to express real addition and multiplication, and demonstrated a number of meta-level properties of this and related languages. Davis, Gotts, and Cohn [6] showed that the constraint language with the primitives EC, PP, and Convex is expressive enough to distinguish any two regions that are not affine transformations of one another; and that it is decidable, but of the same difficulty as solving a set of algebraic inequalities over the reals.

Bennett et al. [2] studied the first-order language over regular regions with predicates $\mathrm{P}(x, y)$ and $\operatorname{Sphere}(x)$. (It is easily shown that this is mutually definable with our structure $\langle\mathcal{U}$,Closer $\rangle$ as long as the universe $\mathcal{U}$ contains spheres.) In a further paper [3] they studied the application of this language to representing rigid body motion. Borgo et al. [4] considered the first order language over regions with the single predicate $\mathrm{P}(x, y)$; the two predicates $\mathrm{P}(x, y)$ and $\mathrm{SC}(x)$ ( $x$ is strongly connected); and the three predicates $\mathrm{P}(x, y), \mathrm{SC}(x)$ and Sphere $(x)$.

Muller [14], systematizing the famous proposal of Hayes [11], constructs a first-order language over four-dimensional regions of space time.

\section{Open Problems}

The most important open problem is the expressivity of the structure $\langle\mathcal{U}, C\rangle$. The results of Schaefer and Stefankovic [19] characterize its complexity, but do not address its expressivity as a geometric language. Pratt and Schoop [17] give strong results on expressivity, but not a complete categorization.

Another interesting problem would be to find general conditions in which analogues of Theorems 1 and 3 apply. Let $\Phi_{1} \ldots \Phi_{m}$ be a collection of relations over closed regions in the plane, and let $\mathcal{U}$ be a collection of closed regions in the plane. Let $\mathcal{H}$ be the group of homeomorphisms of the plane to itself such that $\Phi_{1} \ldots \Phi_{m}$ and $\mathcal{U}$ are invariant under $\mathcal{H}$. What conditions on the $\Phi_{i}$ and $\mathcal{U}$ are sufficient to determine that every analytical relation $\Psi$ that is invariant under $\mathcal{H}$ is first-order definable in the structure $\left\langle\mathcal{U}, \Phi_{1} \ldots \Phi_{m}\right\rangle$ ? Theorems 1 and 3 show that this is true in the specific case where $\mathcal{U}$ contains all polygons and the $\Phi$ 's are either $\{\mathrm{C}$, Convex $\}$ or $\{$ Closer $\}$, but perhaps a more general result is attainable. 


\section{References}

[1] B. Bennett. A Categorical Axiomatisation of Region-Based Geometry. Fundamenta Informaticae, 46, 145-158. 2001.

[2] B. Bennett, A.G. Cohn, P. Torini, and S. Hazarika "A foundation for region-based geometry." Proceedings of the Fifteenth European Conference on Artificial Intelligence. 204-208. 2000.

[3] B. Bennett, A.G. Cohn, P. Torini, and S. Hazarika. Describing rigid-body motion in a qualitative theory of spatial regions. Proceedings, Seventeenth National Conference on Artificial Intelligence, 503-509. 2000.

[4] S. Borgo, N. Guarino, and C. Masolo. A Pointless Theory of Space Based on Strong Connection and Congruence. Fifth International Conference on Principles of Knowledge Representation and Reasoning. 220-229. 1996.

[5] B.L. Clarke. A Calculus of Individuals Based on "Connection". Notre Dame Journal of Formal Logic, 23(3), 204-218. 1981.

[6] E. Davis, N.M. Gotts, and A.G. Cohn. Constraint Networks of Topological Relations and Convexity. CONSTRAINTS, 4(3), 241-280. 1999.

[7] T. de Laguna. Point, line, and surface as sets of solids. Journal of Philosophy, 19, 449-461. 1922.

[8] C. Dornheim. Undecidability of plane polygonal mereotopology. Sixth International Conference on Principles of Knowledge Representation and Reasoning. 342-353. 1998.

[9] N.M. Gotts. How far can we 'C'? Defining a doughnut using connection alone. Fourth International Conference on Principles of Knowledge Representation and Reasoning. 246-257. 1994.

[10] A. Grzegorczyk. Undecidability of some topological theories. Fundamenta Mathematicae. 38, 137-152. 1951.

[11] P. Hayes. The Naive Physics Manifesto. In Expert Systems in the Microelectronic Age. D. Michie (ed.) Edinburgh University Press, Edinburgh, 1979.

[12] P.G. Hinman. Recursion Theoretic Hierarchies. Springer-Verlag, Berlin. 1978.

[13] W. Hodges, Model Theory, Cambridge University Press, Cambridge, 1993.

[14] P. Muller. A qualitative theory of motion based on spatio-temporal primitives. Sixth International Conference on Principles of Knowledge Representation and Reasoning. 131-141. 1998.

[15] B. Nebel. Computational properties of qualitative spatial reasoning: First results. In KI-95: Advanced in Artificial Intelligence, I. Wachsmuth, C-R. Rollinger, and W. Brauer (ed.s), LCNS No. 981, Springer-Verlag, 233-244, 1995.

[16] I. Pratt. First-Order Qualitative Spatial Representation Languages with Convexity. Spatial Cognition and Computation, 1, 181-204. 1999.

[17] I. Pratt and D. Schoop. Expressivity in polygonal, plane mereotopology. Journal of Symbolic Logic. 65(2), 822-838. 2000.

[18] D.A. Randell, Z. Cui, and A.G. Cohn. A spatial logic based on regions and connection. Third International Conference on Principles of Knowledge Representation and Reasoning. 165-176. 1992. 
[19] M. Schaefer and D. Stefankovic. Decidability of string graphs. Journal of Computer and System Sciences. 68, 319-334. 2004.

[20] A. Tarski. Foundations of the Geometry of Solids. In A. Tarski, Logic, Semantics, Metamathematics. Oxford Clarendon Press. 1956.

[21] A.N. Whitehead. Process and Reality. Macmillan, New York. 1929. 